\title{
长度偏差右删失数据下分位数回归的估计 方程方法
}

\author{
马慧娟 ${ }^{(1)}$, 范彩云 ${ }^{(2)(3 *}$ ，周勇 ${ }^{3(4)}$ \\ (1) 中国科学技术大学管理学院统计与金融系, 合肥 230026 ; \\ (2) 上海对外经贸大学商务信息学院, 上海 201620; \\ (3) 上海财经大学统计与管理学院, 上海 200433; \\ (4) 中国科学院数学与系统科学研究院应用数学研究所, 北京 100190 \\ E-mail: mhj@mail.ustc.edu.cn, fancaiyun2010@163.com,yzhou@amss.ac.cn
}

收稿日期: 2014-06-16; 接受日期: 2014-09-27; * 通信作者

国家自然科学基金 (批准号: 71331006 和 71271128)、中国科学院重点实验室 (批准号: 2008Dp173182)、国家数学与交叉科学中 心、长江学者和教育部创新团队发展计划 (批准号: IRT13077)、上海财经大学 211 工程四期和上海财经大学研究生创新基金 (批准 号: CXJJ-2013-449) 资助项目

摘要 本文首先建立左截断右删失数据下的一般分位数回归方法. 当截断变量服从均匀分布时, 左截 断右删失数据变成长度偏差右删失数据。长度偏差数据因其特殊性, 提供了更多的信息. 当把适用于 左截断右删失数据的一般方法用到长度偏差右删失数据时, 得到的估计量并不有效, 这是因为它们没 有利用该数据的特殊结构. 为了提高效率, 本文提出复合估计方程方法来解决长度偏差右删失数据下 的分位数回归问题,这种方法并不需要估计删失变量的分布. 所提出的估计方程可以通过一个求 $L_{1}$ 型 凸函数最小值的简单算法来求解. 本文用经验过程和随机积分的技巧建立了所提出估计量的一致相合 性和弱收敛性. 随机模拟验证了所提出方法在有限样本时的表现, 并且给出了实例分析.

关键词 左截断 右删失 长度偏差抽样 分位数回归 经验过程

MSC (2010) 主题分类 $62 \mathrm{~N} 02$

\section{1 引言}

在生存分析中, 有许多文献研究右删失数据. 除了提前离开实验或者其他原因导致右删失以外, 我们所收集到的数据有时也是左截断的, 例如, 美国 Channing House 数据 (Channing House data)、 加拿大健康年龄中心的老年痴呆数据 (Canadian Study of Health and Aging dementia data) 和西班牙 失业数据 (Spain unemployment data) 等. 特别地, 在许多应用中, 事件的发生次数被假设服从一个 Poisson 过程 ${ }^{[1,2]}$, 也就是说, 事件发生次数随着时间的变化是稳定的. 在这个稳定的假设下, 左截断变 量服从均匀分布, 并且生存时间被抽样的概率和它的长度成正比. 当截断变量服从均匀分布时, 左截断 的生存时间称作长度偏差数据 ${ }^{[3,4]}$. 事实上, 前面提到的加拿大老年痴呆数据和西班牙失业数据都可 当作长度偏差数据来处理 ${ }^{[4-9]}$. 美国 Channing House 数据经过简单的处理后也可被看作是长度偏差 数据 ${ }^{[10,11]}$. 
大量的文献研究了左截断右删失数据和它的特殊情形 一 长度偏差右删失数据. 其中一类是不利 用协变量信息而提出非参数的估计方法, 如文献 $[12,13]$. 另一类文献是在考虑协变量的情形下提出各 种半参回归模型. 对一般的左截断数据, 文献 [14] 在 Cox 比例风险模型下提出了估计方法, 而文献 [15] 得到了加性风险模型下的估计量. 当数据为长度偏差且没有删失时, 文献 [16] 对于 Cox 模型提出了估 计方法, 而文献 [17] 对于加速失效 (AFT) 模型提出了两阶段的秩估计方法. 对长度偏差右删失数据, 文献 $[8,9,18]$ 提出了 Cox 模型下的多种估计方法, 文献 [6] 针对半参转移模型和加速失效 (AFT) 模型 给出了多种不同的估计方程. 文献 [19] 在 AFT 模型下提出了 Buckley-James 类型的估计量. 这些流 行的半参模型限制了协变量只影响生存时间的位置而不是形状.

与 AFT 模型比起来, 分位数回归 ${ }^{[20]}$ 在评价协变量对生存时间的影响方面提供了更大的灵活性, 因而在很多应用中引起了广泛的关注. 分位数回归模型是对生存时间的条件分位数直接进行建模, 得 到的结果也更加容易解释. 而且, 分位数回归允许协变量变化时生存分布有不同的尾部, 因而更容易 抓住样本的非齐次性 ${ }^{[21]}$. 许多文献研究的是利用分位数回归方法处理右删失数据, 如文献 [22-25]. 也 有一些文献研究在左截断右删失数据下 ${ }^{[26,27]}$ 和长度偏差右删失数据下 ${ }^{[10,28]}$ 的分位数回归方法. 以 上处理长度偏差右删失数据的分位数研究方法都需要估计删失变量的分布.

本文提出了处理长度偏差右删失数据分位数回归的新方法, 该方法并不需要估计删失变量的分布. 因此, 与文献 $[10,28]$ 比起来, 我们的方法减少了复杂度. 考虑到左截断右删失数据具有鞅结构特征和 利用长度偏差右删失数据可以构造零均值过程, 这些为证明估计量的大样本性质和进行统计推断带来 了很大的便利性. 我们的第二个方法是利用长度偏差数据的辅助信息, 这与文献 [9] 提出的复合似然 方法很类似, 其中复合似然的方法可以追溯到文献 [29-31]. 借助经验过程和随机积分的技巧, 我们建 立了渐近性质, 包括一致相合性和弱收玫性. 与文献 [24] 类似, 通过最小化一系列 $L_{1}$ 型凸函数, 我们 利用 $\mathrm{R}$ 语言中现有的软件包计算出未知参数. 同时, 我们推广文献 [32] 的方法来估计方差.

本文后续内容安排如下: 第 2 节介绍长度偏差右删失数据下分位数回归的估计方法; 由此得到的 回归分位数过程的一致相合性和渐近正态性将在第 3 节中建立; 方差估计的重抽样技巧和模型检验技 巧分别在第 4 和第 5 节给出; 第 6 节报告了所提方法在有限样本下模拟结果的表现; 第 7 节进行了实 例分析; 第 8 节包含一些注记; 附录中给出一些结论的证明.

\section{2 估计方法}

分位数回归是一个对经典线性回归模型有意义的推广. 给定一个 $p \times 1$ 协变量向量 $\boldsymbol{Z}=\left(1, \tilde{\boldsymbol{Z}}^{\mathrm{T}}\right)^{\mathrm{T}}$ 和 $\tau \in[0,1]$, 随机变量 $X$ 的条件分位数定义为

$$
Q_{X}(\tau \mid \boldsymbol{Z})=\inf \{t: \operatorname{Pr}(X \leqslant t \mid \boldsymbol{Z}) \geqslant \tau\} .
$$

用 $T^{0}$ 表示从感兴趣事件开始到终止的时间, $A^{0}$ 表示从事件开始到抽样检测的时间, $T^{0}$ 被 $A^{0}$ 所 截断. 这意味着, 只有当个体的抽样时间发在事件终止之前时, 它才能被观测到. 记观测到的生存时间 和截断时间分别是 $T$ 和 $A$, 则 $(T, A)$ 与 $\left(T^{0}, A^{0}\right) \mid T^{0} \geqslant A^{0}$ 具有相同的联合分布. 由于提前离开实验或 者其他一些原因, 观测到的生存时间通常是右删失的. 因而, 我们观测到的生存时间并不是真实的 $T$, 而是带有删失的 $Y=\min (T, A+C)$, 其中 $C$ 是进入实验后的删失时间. 记 $\tilde{V}=\min (T-A, C)$ 为观测 到的剩余生存时间, 也就是说, 对于未删失的个体, $\tilde{V}=V$; 对于删失的个体, $\tilde{V}=C$.

在给定协变量 $\boldsymbol{Z}$ 时, 生存时间 $T^{0}$ 的条件密度函数、条件生存函数和条件失效率函数分别记作 $f(t \mid \boldsymbol{Z}), S(t \mid \boldsymbol{Z})$ 和 $\lambda(t \mid \boldsymbol{Z})$. 假设 $C$ 与 $(T, A)$ 在给定 $\boldsymbol{Z}$ 时相互独立, 这在许多应用中是合理的. 然 
而注意到, 观测到的生存时间 $T$ 和整体的删失时间 $A+C$ 是相依的, 因为它们具有共同的 $A$. 换句话 讲, 生存时间 $T$ 是有信息删失的. 用 $\widetilde{G}(t \mid \boldsymbol{Z})$ 表示 $C$ 在给定 $\boldsymbol{Z}$ 时的生存函数. 我们假设观测到的数 据 $\left\{\left(Y_{i}, A_{i}, \mathbf{Z}_{i}, \delta_{i}\right), i=1,2, \ldots, n\right\}$ 是 $(Y, A, \mathbf{Z}, \delta)$ 的简单随机样本, 其中 $\delta=I(T \leqslant A+C)$.

本文假设如下模型:

$$
Q_{T^{0}}(\tau \mid \boldsymbol{Z})=\exp \left\{\boldsymbol{Z}^{\mathrm{T}} \boldsymbol{\beta}(\tau)\right\}, \quad \tau \in(0,1)
$$

\section{1 基于鞅的估计方程方法}

当数据没有截断时, 或者等价地, 当 $A=0$ 时, 文献 [24] 基于鞅的估计方程得到了分位数回归模 型. 在给定截断时间 $A$ 的条件下, 文献 [24] 提出的方法可以被简单地推广到左截断右删失数据. 特别 地, $N_{i}(t)=I\left(Y_{i} \leqslant t, \delta_{i}=1\right)$ 表示第 $i$ 个个体是否在时间 $t$ 之前死亡, $Y_{i}^{a}(t)=I\left(A_{i} \leqslant t \leqslant Y_{i}\right)$ 表示第 $i$ 个个体在 $t$ 时刻是否在险. 文献 [33] 中已经证明, 对每个 $i$, 计数过程 $N_{i}(t)$ 可以被唯一分解成

$$
N_{i}(t)=M_{1 i}(t)+\int_{0}^{t} Y_{i}^{a}(u) \lambda\left(u \mid Z_{i}\right) d u,
$$

其中 $M_{1 i}(t)$ 是一个局部可积鞅.

对 $t \geqslant 0$, 因为 $\mathrm{E}\left\{M_{1 i}(t) \mid \boldsymbol{Z}_{i}\right\}=0$, 我们有

$$
\begin{aligned}
\mathrm{E}\{ & \left.\sum_{i=1}^{n} \boldsymbol{Z}_{i}\left(N_{i}\left(\exp \left\{\boldsymbol{Z}_{i}^{\mathrm{T}} \boldsymbol{\beta}_{0}(\tau)\right\}\right)-\int_{0}^{\exp \left\{\boldsymbol{Z}_{i}^{\mathrm{T}} \boldsymbol{\beta}_{0}(\tau)\right\}} Y_{i}^{a}(u) \lambda\left(u \mid \boldsymbol{Z}_{i}\right) d u\right)\right\} \\
& =\mathrm{E}\left\{\sum_{i=1}^{n} \boldsymbol{Z}_{i}\left(N_{i}\left(\exp \left\{\boldsymbol{Z}_{i}^{\mathrm{T}} \boldsymbol{\beta}_{0}(\tau)\right\}\right)-\int_{0}^{\exp \left\{\boldsymbol{Z}_{i}^{\mathrm{T}} \boldsymbol{\beta}_{0}(\tau)\right\}} Y_{i}^{a}(u) d\left[-\log \left(1-F_{T^{0}}\left(u \mid \boldsymbol{Z}_{i}\right)\right)\right]\right)\right\} \\
& =\mathrm{E}\left\{\sum_{i=1}^{n} \boldsymbol{Z}_{i}\left(N_{i}\left(\exp \left\{\boldsymbol{Z}_{i}^{\mathrm{T}} \boldsymbol{\beta}_{0}(\tau)\right\}\right)-\int_{0}^{\tau} Y_{i}^{a}\left(\exp \left\{\boldsymbol{Z}_{i}^{\mathrm{T}} \boldsymbol{\beta}_{0}(u)\right\}\right) d H(u)\right)\right\}=0,
\end{aligned}
$$

其中 $H(x)=-\log (1-x), 0 \leqslant x<1, \boldsymbol{\beta}_{0}(\cdot)$ 是 $\boldsymbol{\beta}(\cdot)$ 的真实值, 第二个方程成立是因为 $\boldsymbol{Z}_{i}^{\mathrm{T}} \boldsymbol{\beta}_{0}(\tau)$ 关于 $\tau$ 的单调性和 $F_{T^{0}}\left(\exp \left\{\boldsymbol{Z}_{i}^{\mathrm{T}} \boldsymbol{\beta}_{0}(u)\right\} \mid \boldsymbol{Z}_{i}\right)=u$. 这启发我们考虑如下的估计方程:

$$
n^{1 / 2} \boldsymbol{S}_{1 n}\{\boldsymbol{\beta}(\tau), \tau\}=0
$$

其中

$$
\boldsymbol{S}_{1 n}(\boldsymbol{b}, \tau)=n^{-1} \sum_{i=1}^{n} \boldsymbol{Z}_{i}\left(N_{i}\left(\exp \left\{\boldsymbol{Z}_{i}^{\mathrm{T}} \boldsymbol{b}\right\}\right)-\int_{0}^{\tau} Y_{i}^{a}\left(\exp \left\{\boldsymbol{Z}_{i}^{\mathrm{T}} \boldsymbol{b}\right\}\right) d H(u)\right) .
$$

记 $\boldsymbol{s}_{1}(\boldsymbol{b}, \tau)=\mathrm{E}\left\{\boldsymbol{S}_{1 n}(\boldsymbol{b}, \tau)\right\}$. 鞅 $M_{1 i}(\cdot)$ 使得 $\boldsymbol{s}_{1}\left\{\boldsymbol{\beta}_{0}(\tau), \tau\right\}=0$. 事实上, 估计方程 (2.2) 可以被用来处理 一般的左截断右删失数据, 也就是使平稳条件不成立的情形.

$\boldsymbol{S}_{1 n}(\boldsymbol{b}, \tau)$ 中的随机积分表示启发我们用格子点估计方法来估计 $\boldsymbol{\beta}_{0}(\tau)$. 特别地, 记 $\tilde{\boldsymbol{\beta}}(\tau)$ 为 $\boldsymbol{\beta}_{0}(\tau)$ 的一个估计, 且是只在格子点 $\mathcal{S}_{L_{n}}=\left\{0=\tau_{0}<\tau_{1}<\cdots<\tau_{L_{n}}=\tau_{U}<1\right\}$ 处有跳跃的分段右连续函数, 其中 $\tau_{U} \in(0,1)$ 是一个满足特定的由于右删失导致的可识别限制的常数. 关于 $\tau_{U}$ 的精确条件将会在 后面给渐近结果时给出. 与文献 $[24,34]$ 类似, 我们仅仅考虑 $\left\{\boldsymbol{\beta}_{0}(\tau), \tau \in\left(0, \tau_{U}\right]\right\}$. 令 $\exp \left\{\boldsymbol{Z}^{\mathrm{T}} \tilde{\boldsymbol{\beta}}(0)\right\}=0$, 这是因为 $0=Q_{T^{0}}(0 \mid \boldsymbol{Z})=\exp \left\{\boldsymbol{Z}^{\mathrm{T}} \boldsymbol{\beta}_{0}(0)\right\}$. 基于 $\tilde{\boldsymbol{\beta}}(\tau)$ 为分段常数的性质, (2.2) 中的积分简化为一个 求和. 因而, 我们可以通过连续的解如下关于 $\boldsymbol{\beta}\left(\tau_{j}\right)$ 的单调估计方程:

$$
n^{-1 / 2} \sum_{i=1}^{n} \boldsymbol{Z}_{i}\left(N_{i}\left(\exp \left\{\boldsymbol{Z}_{i}^{\mathrm{T}} \boldsymbol{\beta}\left(\tau_{j}\right)\right\}\right)-\sum_{k=0}^{j-1} Y_{i}^{a}\left(\exp \left\{\boldsymbol{Z}_{i}^{\mathrm{T}} \tilde{\boldsymbol{\beta}}\left(\tau_{k}\right)\right\}\right)\left\{H\left(\tau_{k+1}\right)-H\left(\tau_{k}\right)\right\}\right)=0
$$


来得到 $\left\{\tilde{\boldsymbol{\beta}}\left(\tau_{j}\right), j=1, \ldots, L_{n}\right\}$. 因为 (2.3) 并不是连续的, 可能并不存在精确的解. 经过一些简单的代 数计算后, 我们发现 $(2.3)$ 的求解问题等价于寻找如下 $L_{1}$ 型凸函数的最小值:

$$
\begin{aligned}
l_{1 j}(\boldsymbol{h})= & \sum_{i=1}^{n}\left|\delta_{i} \log Y_{i}-\delta_{i} \boldsymbol{h}^{\mathrm{T}} \boldsymbol{Z}_{i}\right|+\left|M-\boldsymbol{h}^{\mathrm{T}} \sum_{l=1}^{n}\left(-\delta_{l} \boldsymbol{Z}_{l}\right)\right| \\
& +\left|M-\boldsymbol{h}^{\mathrm{T}} \sum_{r=1}^{n}\left(2 \boldsymbol{Z}_{r} \sum_{k=0}^{j-1} Y_{r}^{a}\left(\exp \left\{\boldsymbol{Z}_{r}^{\mathrm{T}} \tilde{\boldsymbol{\beta}}\left(\tau_{k}\right)\right\}\right)\left\{H\left(\tau_{k+1}\right)-H\left(\tau_{k}\right)\right\}\right)\right|,
\end{aligned}
$$

其中 $M$ 是一个非常大的数, 而 $j=1, \ldots, L_{n}$. 这个最小值问题可以通过标准的统计软件容易地解决, 例如, $\mathrm{R}$ 软件包 quantreg 中的 $r q()$ 函数.

\section{2 复合估计方程方法}

因为长度偏差数据是一种特殊的左截断右删失数据, 上面提出的条件估计方程 (2.2) 可以用到长 度偏差右删失数据上. 当用上面的这个方法时, 得到的估计量并不是有效的, 因为它们并没有利用到 长度偏差右删失数据的特殊结构. 本小节提出更加有效的复合估计方程方法来提高效率.

根据文献 [9], 当数据没有删失时, 随机变量 $A$ 和 $V=T-A$ 具有相同的边际密度函数. 当数据存 在删失时, $A$ 和 $\tilde{V}=\min (T-A, C)$ 的联合分布因为右删失的出现不再是可交换的. 然而, 在给定观测 的生存时间未删失时, 给定 $A$ 下 $\tilde{V}$ 的分布和给定 $\tilde{V}$ 时 $A$ 的分布是相同的. 它们利用这个性质通过 用复合条件似然函数的方法来得到 Cox 模型中回归参数和相应累积风险函数的估计. 类似地, 本文利 用复合估计方程的方法, 构造零均值的随机过程 $M_{2 i}(t)$ :

$$
M_{2 i}(t)=N_{i}(t)-\int_{0}^{t} \delta_{i} I\left(\tilde{V}_{i} \leqslant t \leqslant Y_{i}\right) \lambda\left(u \mid \boldsymbol{Z}_{i}\right) d u .
$$

事实上, 容易证明

$$
E\left[d N_{i}(t) \mid \boldsymbol{Z}_{i}\right]=E\left[\delta_{i} I\left(\tilde{V}_{i} \leqslant t \leqslant Y_{i}\right) \lambda\left(t \mid \boldsymbol{Z}_{i}\right) d t\right]=\frac{f\left(t \mid \boldsymbol{Z}_{i}\right)}{\mu\left(\boldsymbol{Z}_{i}\right)} \omega_{c}(t \mid \boldsymbol{Z}) d t,
$$

其中

$$
\omega_{c}(t \mid \boldsymbol{Z})=\int_{0}^{t} \widetilde{G}\left(u \mid \boldsymbol{Z}_{i}\right) d u,
$$

$\widetilde{G}\left(u \mid \boldsymbol{Z}_{i}\right)$ 是给定协变量 $\boldsymbol{Z}_{i}$ 时 $C$ 的生存函数.

为了表述更加简洁, 令

$$
M_{i}(t)=\frac{1}{2}\left[M_{1 i}(t)+M_{2 i}(t)\right]=N_{i}(t)-\int_{0}^{t} R_{i}(u) \lambda(u \mid \boldsymbol{Z}) d u,
$$

其中

$$
R_{i}(t)=\frac{1}{2}\left\{I\left(A_{i} \leqslant t \leqslant Y_{i}\right)+\delta_{i} I\left(\tilde{V}_{i} \leqslant t \leqslant Y_{i}\right)\right\} .
$$

因为对 $t \geqslant 0, \mathrm{E}\left\{M_{i}(t) \mid \boldsymbol{Z}_{i}\right\}=0$, 类似地, 我们有

$$
\begin{aligned}
\mathrm{E}\{ & \left.\sum_{i=1}^{n} \boldsymbol{Z}_{i}\left(N_{i}\left(\exp \left\{\boldsymbol{Z}_{i}^{\mathrm{T}} \boldsymbol{\beta}_{0}(\tau)\right\}\right)-\int_{0}^{\exp \left\{\boldsymbol{Z}_{i}^{\mathrm{T}} \boldsymbol{\beta}_{0}(\tau)\right\}} R_{i}(u) \lambda\left(u \mid \boldsymbol{Z}_{i}\right) d u\right)\right\} \\
& =\mathrm{E}\left\{\sum_{i=1}^{n} \boldsymbol{Z}_{i}\left(N_{i}\left(\exp \left\{\boldsymbol{Z}_{i}^{\mathrm{T}} \boldsymbol{\beta}_{0}(\tau)\right\}\right)-\int_{0}^{\exp \left\{\boldsymbol{Z}_{i}^{\mathrm{T}} \boldsymbol{\beta}_{0}(\tau)\right\}} R_{i}(u) d\left[-\log \left(1-F_{T^{0}}\left(u \mid \boldsymbol{Z}_{i}\right)\right)\right]\right)\right\}
\end{aligned}
$$

1984 


$$
=\mathrm{E}\left\{\sum_{i=1}^{n} \boldsymbol{Z}_{i}\left(N_{i}\left(\exp \left\{\boldsymbol{Z}_{i}^{\mathrm{T}} \boldsymbol{\beta}_{0}(\tau)\right\}\right)-\int_{0}^{\tau} R_{i}\left(\exp \left\{\boldsymbol{Z}_{i}^{\mathrm{T}} \boldsymbol{\beta}_{0}(u)\right\}\right) d H(u)\right)\right\}=0
$$

因而, 我们考虑估计方程

$$
n^{1 / 2} \boldsymbol{S}_{n}\{\boldsymbol{\beta}(\tau), \tau\}=0,
$$

其中

$$
\boldsymbol{S}_{n}(\boldsymbol{b}, \tau)=n^{-1} \sum_{i=1}^{n} \boldsymbol{Z}_{i}\left(N_{i}\left(\exp \left\{\boldsymbol{Z}_{i}^{\mathrm{T}} \boldsymbol{b}\right\}\right)-\int_{0}^{\tau} R_{i}\left(\exp \left\{\boldsymbol{Z}_{i}^{\mathrm{T}} \boldsymbol{b}\right\}\right) d H(u)\right) .
$$

令 $\boldsymbol{s}(\boldsymbol{b}, \tau)=\mathrm{E}\left\{\boldsymbol{S}_{n}(\boldsymbol{b}, \tau)\right\}$, 由于零均值结构, 故有 $\boldsymbol{s}\left\{\boldsymbol{\beta}_{0}(\tau), \tau\right\}=0$.

$\boldsymbol{S}_{n}(\boldsymbol{b}, \tau)$ 的随机积分表达式启发我们用一个类似上面的方法来估计 $\boldsymbol{\beta}_{0}(\tau)$. 记该估计量为 $\hat{\boldsymbol{\beta}}(\tau)$, 一个仅在格子点 $\mathcal{S}_{L_{n}}$ 上有跳跃的右连续分段常数函数. 我们也仅仅考虑 $\left\{\boldsymbol{\beta}_{0}(\tau), \tau \in\left(0, \tau_{U}\right]\right\}$. 可以通 过连续地求解如下关于 $\boldsymbol{\beta}\left(\tau_{j}\right)$ 的单调估计方程来得到 $\left\{\hat{\boldsymbol{\beta}}\left(\tau_{j}\right), j=1, \ldots, L_{n}\right\}$ :

$$
n^{-1 / 2} \sum_{i=1}^{n} \boldsymbol{Z}_{i}\left(N_{i}\left(\exp \left\{\boldsymbol{Z}_{i}^{\mathrm{T}} \boldsymbol{\beta}\left(\tau_{j}\right)\right\}\right)-\sum_{k=0}^{j-1} R_{i}\left(\exp \left\{\boldsymbol{Z}_{i}^{\mathrm{T}} \hat{\boldsymbol{\beta}}\left(\tau_{k}\right)\right\}\right)\left\{H\left(\tau_{k+1}\right)-H\left(\tau_{k}\right)\right\}\right),
$$

其中 $\exp \left\{\boldsymbol{Z}^{\mathrm{T}} \hat{\boldsymbol{\beta}}(0)\right\}=0 .(2.5)$ 的求解问题等价于求如下 $L_{1}$ 型凸函数的最小值,

$$
\begin{aligned}
l_{j}(\boldsymbol{h})= & \sum_{i=1}^{n}\left|\delta_{i} \log Y_{i}-\delta_{i} \boldsymbol{h}^{\mathrm{T}} \boldsymbol{Z}_{i}\right|+\left|M-\boldsymbol{h}^{\mathrm{T}} \sum_{l=1}^{n}\left(-\delta_{l} \boldsymbol{Z}_{l}\right)\right| \\
& +\left|M-\boldsymbol{h}^{\mathrm{T}} \sum_{r=1}^{n}\left(2 \boldsymbol{Z}_{r} \sum_{k=0}^{j-1} R_{r}\left(\exp \left\{\boldsymbol{Z}_{r}^{\mathrm{T}} \hat{\boldsymbol{\beta}}\left(\tau_{k}\right)\right\}\right)\left\{H\left(\tau_{k+1}\right)-H\left(\tau_{k}\right)\right\}\right)\right|
\end{aligned}
$$

其中 $M$ 是一个很大的数, 而 $j=1, \ldots, L_{n}$. 上面的最小化问题可以用 $\mathrm{R}$ 程序包 quantreg 中的 $r q()$ 函 数求解.

\section{3 渐近性质}

类似文献 [24] 中的证明方法, 可以得到 $\tilde{\boldsymbol{\beta}}(\cdot)$ 的大样本性质. 本节给出所提出估计量 $\hat{\boldsymbol{\beta}}(\cdot)$ 的一致 相合性和弱收敛性.

为了表述正则条件, 我们需要更多的记号. 定义

$$
\begin{aligned}
\tilde{F}(t \mid \boldsymbol{Z}) & =\operatorname{Pr}(Y \leqslant t, \delta=1 \mid \boldsymbol{Z}), \quad \tilde{f}(t \mid \boldsymbol{Z})=\frac{d \tilde{F}(t \mid \boldsymbol{Z})}{d t}, \\
\bar{F}(t \mid \boldsymbol{Z}) & =\frac{1}{2}[\operatorname{Pr}(A \leqslant t \leqslant Y \mid \boldsymbol{Z})+\operatorname{Pr}(\tilde{V} \leqslant t \leqslant Y \mid \boldsymbol{Z})] \\
& =\frac{1}{2}\left[F_{A}(t \mid \boldsymbol{Z})+F_{\tilde{V}}(t \mid \boldsymbol{Z})\right]-F_{Y}(t \mid \boldsymbol{Z}),
\end{aligned}
$$

其中 $F_{X}(t \mid \boldsymbol{Z})=\operatorname{Pr}(X \leqslant t \mid \boldsymbol{Z}), \bar{f}(t \mid \boldsymbol{Z})=d \bar{F}(t \mid \boldsymbol{Z}) / d t$. 对 $\boldsymbol{b} \in \mathbb{R}^{p}$, 定义

$$
\begin{aligned}
& \tilde{\mu}(\boldsymbol{b})=\mathrm{E}\left[\boldsymbol{Z} N\left\{\exp \left(\boldsymbol{Z}^{\mathrm{T}} \boldsymbol{b}\right)\right\}\right], \\
& B(\boldsymbol{b})=\mathrm{E}\left[\boldsymbol{Z}^{\otimes 2} \tilde{f}\left\{\exp \left(\boldsymbol{Z}^{\mathrm{T}} \boldsymbol{b}\right) \mid \boldsymbol{Z}\right\} \exp \left(\boldsymbol{Z}^{\mathrm{T}} \boldsymbol{b}\right)\right], \\
& J(\boldsymbol{b})=\mathrm{E}\left[\boldsymbol{Z}^{\otimes 2} \bar{f}\left\{\exp \left(\boldsymbol{Z}^{\mathrm{T}} \boldsymbol{b}\right) \mid \boldsymbol{Z}\right\} \exp \left(\boldsymbol{Z}^{\mathrm{T}} \boldsymbol{b}\right)\right] .
\end{aligned}
$$


用 $\mathcal{Z}$ 表示协变量空间,

$$
\mathcal{O}(\boldsymbol{\beta}, d)=\left\{\boldsymbol{b} \in \mathbb{R}^{p}: \inf _{\tau \in\left(0, \tau_{U}\right]}\|\tilde{\mu}(\boldsymbol{b})-\tilde{\mu}(\boldsymbol{\beta}(\tau))\| \leqslant d\right\} .
$$

对一个向量 $\boldsymbol{u}$, 用 $\boldsymbol{u}^{\otimes 2}$ 表示 $\boldsymbol{u} \boldsymbol{u}^{\mathrm{T}},\|\boldsymbol{u}\|$ 表示 $\boldsymbol{u}$ 的 Euclid 范数. 本文所需的正则条件表示如下:

(C1) $\mathcal{Z}$ 是紧集, 也就是说, $\sup _{i}\left\|\boldsymbol{Z}_{i}\right\|<\infty$;

$(\mathrm{C} 2)$ 对每个 $\boldsymbol{Z}, \tilde{f}(t \mid \boldsymbol{Z})$ 和 $\bar{f}(t \mid \boldsymbol{Z})$ 关于 $t$ 是连续的;

(C3) $\tilde{f}(t \mid \boldsymbol{Z})$ 和 $\bar{f}(t \mid \boldsymbol{Z})$ 一致关于 $t$ 和 $\boldsymbol{Z}$ 是有界的;

(C4) 存在一个常数 $d_{0}>0$, 对于所有的 $\boldsymbol{b} \in \mathcal{O}\left(\boldsymbol{\beta}_{0}, d_{0}\right)$, 使得 $\tilde{f}\left\{\exp \left(\boldsymbol{Z}^{\mathrm{T}} \boldsymbol{b}\right) \mid \boldsymbol{Z}\right\}>0$;

(C5) $\sup _{\boldsymbol{b} \in \mathcal{O}\left(\boldsymbol{\beta}_{0}, d_{0}\right)}\left\|B(\boldsymbol{b})^{-1} J(\boldsymbol{b})\right\|<\infty$.

根据条件 $(\mathrm{C} 1)$ 和 $(\mathrm{C} 2), \tilde{\mu}\left\{\boldsymbol{\beta}_{0}(\tau)\right\}$ 是一个关于 $\tau$ 的 Lipschitz 函数 ${ }^{[34]}$. 然后, 我们有如下的定理.

定理 1 假设正则条件 $(\mathrm{C} 1)-(\mathrm{C} 5)$ 成立, 如果

$$
\lim _{n \rightarrow \infty}\left\|\mathcal{S}_{L_{n}}\right\|=0,
$$

则随着 $n \rightarrow \infty, \sup _{\tau \in\left[\nu, \tau_{U}\right]}\left\|\hat{\boldsymbol{\beta}}(\tau)-\boldsymbol{\beta}_{0}(\tau)\right\|$ 依概率收玫到 0 , 其中 $0<\nu<\tau_{U}$,

$$
\left\|\mathcal{S}_{L_{n}}\right\|=\max \left\{\tau_{j}-\tau_{j-1} ; j=1, \ldots, L_{n}\right\}
$$

表示 $\mathcal{S}_{L_{n}}$ 的大小.

定理 2 假设正则条件 $(\mathrm{C} 1)-(\mathrm{C} 5)$ 成立, 如果

$$
\lim _{n \rightarrow \infty} n^{1 / 2}\left\|\mathcal{S}_{L_{n}}\right\|=0
$$

则对 $\tau \in\left[\nu, \tau_{U}\right], n^{1 / 2}\left\{\hat{\boldsymbol{\beta}}(\tau)-\boldsymbol{\beta}_{0}(\tau)\right\}$ 弱收玫到一个零均值的 Gauss 过程, 其中 $0<\nu<\tau_{U}$.

\section{4 方差估计的重抽样方法}

回归系数估计量的极限方差很复杂, 因为它们包含未知的密度函数, 这在有限样本下是很难估计 的. 本节提出一个简单的重抽样方法来估计 $\tilde{\boldsymbol{\beta}}\left(\tau_{*}\right)$ 和 $\hat{\boldsymbol{\beta}}\left(\tau_{*}\right)$ 的方差, 这种方法推广了文献 [32] 中提出 的极小化扰动技巧.

首先介绍 $\tilde{\boldsymbol{\beta}}\left(\tau_{*}\right)$ 的方差估计. 假设 $\zeta_{1}, \ldots, \zeta_{n}$ 是从均值为 1 方差为 1 的非负已知分布中抽出的独 立随机样本, 例如, 从均值为 1 的指数分布中抽出的样本. 定义 $\tilde{\boldsymbol{\beta}}^{*}(\tau)$ 为仅在 $\tau_{j}, j=1, \ldots, L_{n}$ 处有跳 跃的右连续分段函数. 对每个 $j \in\left\{1, \ldots, L_{n}\right\}$, 我们考虑关于 $\boldsymbol{h}$ 的 $l_{1 j}(\boldsymbol{h})$ 的随机扰动,

$$
l_{1 j}^{*}(\boldsymbol{h})=\sum_{i=1}^{n}\left|\zeta_{i} \delta_{i} \log Y_{i}-\zeta_{i} \delta_{i} \boldsymbol{h}^{\mathrm{T}} \boldsymbol{Z}_{i}\right|+\left|M-\boldsymbol{h}^{\mathrm{T}} \sum_{l=1}^{n}\left(-\zeta_{l} \delta_{l} \boldsymbol{Z}_{l}\right)\right|+\left|M-\boldsymbol{h}^{\mathrm{T}} \sum_{r=1}^{n}\left(2 \zeta_{r} \boldsymbol{Z}_{r} \sum_{k=0}^{j-1} Y_{r}^{a}\right)\right|,
$$

其中 $\exp \left\{\boldsymbol{Z}_{i}^{\mathrm{T}} \tilde{\boldsymbol{\beta}}^{*}(0)\right\}=0, i=1, \ldots, n, \tilde{l}_{1 j}(\boldsymbol{h})$ 中的 $\tilde{\boldsymbol{\beta}}^{*}\left(\tau_{k}\right)$ 是通过连续的极小化 $\tilde{l}_{1 k}(\boldsymbol{h}), k=1, \ldots, j-1$, $j \geqslant 2$ 来得到的. 把观测数据固定, 重复地产生随机变量 $\left\{\zeta_{1}, \ldots, \zeta_{n}\right\}$ 来得到大量 $\tilde{\boldsymbol{\beta}}^{*}(\tau)$ 的样本实 现, 记它们为 $\left\{\tilde{\boldsymbol{\beta}}_{r}^{*}(\tau)\right\}_{r=1}^{B}$. 与文献 [24] 类似, 作为在 $\tau \in\left[\nu, \tau_{U}\right]$ 上的随机过程, 在给定观测数据时, $n^{1 / 2}\left\{\tilde{\boldsymbol{\beta}}^{*}(\tau)-\tilde{\boldsymbol{\beta}}(\tau)\right\}$ 的条件分布和 $n^{1 / 2}\left\{\tilde{\boldsymbol{\beta}}(\tau)-\boldsymbol{\beta}_{0}(\tau)\right\}$ 的无条件分布是渐近等价的. 对于固定的 $\tau_{*} \in$ $\left(0, \tau_{U}\right), \tilde{\boldsymbol{\beta}}\left(\tau_{*}\right)$ 的方差可以由 $\left\{\tilde{\boldsymbol{\beta}}_{r}^{*}\left(\tau_{*}\right)\right\}_{r=1}^{B}$ 的样本方差来估计. $\boldsymbol{\beta}\left(\tau_{*}\right)$ 的置信区间可以通过 $\tilde{\beta}^{*}\left(\tau_{*}\right)$ 的正 态逼近或者经验分位数来建立. 
$\hat{\boldsymbol{\beta}}\left(\tau_{*}\right)$ 的方差估计方法和 $\tilde{\boldsymbol{\beta}}\left(\tau_{*}\right)$ 的一样, 除了把 $l_{1 j}^{*}(\boldsymbol{h})$ 用

$$
\begin{aligned}
l_{j}^{*}(\boldsymbol{h})= & \sum_{i=1}^{n}\left|\zeta_{i} \delta_{i} \log Y_{i}-\zeta_{i} \delta_{i} \boldsymbol{h}^{\mathrm{T}} \boldsymbol{Z}_{i}\right|+\left|M-\boldsymbol{h}^{\mathrm{T}} \sum_{l=1}^{n}\left(-\zeta_{l} \delta_{l} \boldsymbol{Z}_{l}\right)\right| \\
& +\left|M-\boldsymbol{h}^{\mathrm{T}} \sum_{r=1}^{n}\left(2 \zeta_{r} \boldsymbol{Z}_{r} \sum_{k=0}^{j-1} R_{r}\left(\exp \left\{\boldsymbol{Z}_{r}^{\mathrm{T}} \hat{\boldsymbol{\beta}}^{*}\left(\tau_{k}\right)\right\}\right)\left\{H\left(\tau_{k+1}\right)-H\left(\tau_{k}\right)\right\}\right)\right|
\end{aligned}
$$

替代外. 也就是说, 为了得到 $\hat{\boldsymbol{\beta}}\left(\tau_{*}\right)$ 的方差估计, 我们生成随机变量 $\left\{\zeta_{1}, \ldots, \zeta_{n}\right\}$, 最小化 $l_{j}^{*}(\boldsymbol{h})$ 得到 $\left\{\hat{\boldsymbol{\beta}}_{r}^{*}\left(\tau_{*}\right)\right\}_{r=1}^{B}$. 在附录 D 中, 我们将会证明, 作为在 $\tau \in\left[\nu, \tau_{U}\right]$ 上的随机过程, 在给定观测数据时, $n^{1 / 2}\left\{\hat{\boldsymbol{\beta}}^{*}(\tau)-\hat{\boldsymbol{\beta}}(\tau)\right\}$ 的条件分布和 $n^{1 / 2}\left\{\hat{\boldsymbol{\beta}}(\tau)-\boldsymbol{\beta}_{0}(\tau)\right\}$ 的无条件分布是渐近等价的. $\hat{\boldsymbol{\beta}}\left(\tau_{*}\right)$ 的方差和置 信区间可以类似地得到.

\section{5 模型检验方法}

条件估计方程 $(2.2)$ 具有鞅结构, 我们可以用鞅残差和它们的变换来检验模型 $(2.1)^{[35]}$. 考虑一类 简单的随机过程

$$
Q(\tau)=n^{-1 / 2} \sum_{i=1}^{n} q\left(\boldsymbol{Z}_{i}\right) M_{1 i}(\tau ; \tilde{\boldsymbol{\beta}})
$$

其中 $q(\cdot)$ 是一个已知的有界函数,

$$
M_{1 i}(\tau ; \boldsymbol{\beta})=N_{i}\left(\exp \left\{\boldsymbol{Z}_{i}^{\mathrm{T}} \boldsymbol{\beta}(\tau)\right\}\right)-\int_{0}^{\tau} Y_{i}^{a}\left(\exp \left\{\boldsymbol{Z}_{i}^{\mathrm{T}} \boldsymbol{\beta}(u)\right\}\right) d H(u) .
$$

假设模型 (2.1) 是正确的, 类似文献 [24] 的方法可以证明, $Q(\tau)$ 弱收玫到一个零均值的 Gauss 过程. 它的分布可以被

$$
Q^{*}(\tau)=n^{-1 / 2} \sum_{i=1}^{n} q\left(\boldsymbol{Z}_{i}\right) M_{1 i}(\tau ; \tilde{\boldsymbol{\beta}})\left(1-\zeta_{i}\right)+n^{-1 / 2} \sum_{i=1}^{n} q\left(\boldsymbol{Z}_{i}\right)\left\{M_{1 i}\left(\tau ; \tilde{\boldsymbol{\beta}}^{*}\right)-M_{1 i}(\tau ; \tilde{\boldsymbol{\beta}})\right\}
$$

近似逼近得到. 和之前一样, 固定 $Q^{*}(\cdot)$ 中的观测数据 $\left\{A_{i}, Y_{i}, \delta_{i}, \boldsymbol{Z}_{i}\right\}_{i=1}^{n}$. 为了逼近零假设下 $Q(\cdot)$ 的分 布, 通过重复生成 $\left\{\zeta_{i}, i=1, \ldots, n\right\}$, 我们可得到大量 $Q(\cdot)$ 的样本实现. 和 $Q^{*}(\cdot)$ 的表现相比, $Q(\cdot)$ 的 不寻常的表现说明模型拟合不好. 在模型 (2.1) 下, 因为 $Q(\tau)$ 的期望表现是在 0 附近扰动, 上确界统 计量 $\sup _{\tau \in[l, u]}|Q(\tau)|$ 将是拟合优度的一个自然的数值度量, 其中 $0<l<u<\tau_{U}$. 超过 $\sup _{\tau \in[l, u]}|Q(\tau)|$ 的 $\sup _{\tau \in[l, u]}\left|Q^{*}(\tau)\right|$ 中的经验比例值可以逼近 $p$ 值.

复合估计方程 (2.4) 具有零均值随机过程的结构, 我们可以应用残差和它们的变换来检验模 型 (2.1) (参见文献 [36]). 考虑

$$
K(\tau)=n^{-1 / 2} \sum_{i=1}^{n} q\left(\boldsymbol{Z}_{i}\right) M_{i}(\tau ; \hat{\boldsymbol{\beta}}),
$$

其中

$$
M_{i}(\tau ; \boldsymbol{\beta})=N_{i}\left(\exp \left\{\boldsymbol{Z}_{i}^{\mathrm{T}} \boldsymbol{\beta}(\tau)\right\}\right)-\int_{0}^{\tau} R_{i}\left(\exp \left\{\boldsymbol{Z}_{i}^{\mathrm{T}} \boldsymbol{\beta}(u)\right\}\right) d H(u) .
$$

我们将在附录 D 证明, 假设模型正确时, $K(\tau)$ 弱收玫到一个零均值的 Gauss 过程, 它的分布可以用 


$$
K^{*}(\tau)=n^{-1 / 2} \sum_{i=1}^{n} q\left(\boldsymbol{Z}_{i}\right) M_{i}(\tau ; \hat{\boldsymbol{\beta}})\left(1-\zeta_{i}\right)+n^{-1 / 2} \sum_{i=1}^{n} q\left(\boldsymbol{Z}_{i}\right)\left\{M_{i}\left(\tau ; \hat{\boldsymbol{\beta}}^{*}\right)-M_{i}(\tau ; \hat{\boldsymbol{\beta}})\right\}
$$

来逼近. 在 $K^{*}(\cdot)$ 中, 令 $\zeta_{i}$ 随机而 $\left\{A_{i}, Y_{i}, \delta_{i}, \boldsymbol{Z}_{i}\right\}_{i=1}^{n}$ 固定. 通过重复产生 $\left\{\zeta_{i}, i=1, \ldots, n\right\}$, 我们得到 $K^{*}(\cdot)$ 的样本实现值来逼近 $K(\cdot)$ 在零假设下的分布. 我们也用上确界统计量 $\sup _{\tau \in[l, u]}|K(\tau)|$ 来检验 原假设, 其中 $0<l<u<\tau_{U}$. 超过 $\sup _{\tau \in[l, u]}|K(\tau)|$ 的 $\sup _{\tau \in[l, u]}\left|K^{*}(\tau)\right|$ 中的经验比例值可以用来逼 近 $p$ 值. 注意到, 文献 [24] 中讨论的假设检验问题和两阶段推断问题也不难得到.

\section{6 随机模拟}

在本节中, 为了检验所提出方法在有限样本下的表现, 我们给出随机模拟结果, 见表 1 和 2 . 我们 只生成长度偏差右删失数据, 并分别用两种方法来分析它, 同时, 我们也给出忽略长度偏差结构时的 模拟结果, 见表 3 和 4.

表 1 同方差模型 $(\gamma=0)$ 下的随机模拟结果

\begin{tabular}{|c|c|c|c|c|c|c|c|c|}
\hline \multirow[b]{2}{*}{ 删失率 } & \multirow[b]{2}{*}{$\tau$} & & \multicolumn{3}{|c|}{ 最小化 $l_{1 j}$} & \multicolumn{3}{|c|}{ 最小化 $l_{j}$} \\
\hline & & & $\tilde{\beta}^{(0)}$ & $\tilde{\beta}^{(1)}$ & $\tilde{\beta}^{(2)}$ & $\hat{\beta}^{(0)}$ & $\hat{\beta}^{(1)}$ & $\hat{\beta}^{(2)}$ \\
\hline \multirow[t]{12}{*}{$20 \%$} & 0.25 & Bias & -0.025 & -0.001 & -0.009 & -0.025 & -0.001 & -0.008 \\
\hline & & $\mathrm{SD}$ & 0.069 & 0.039 & 0.081 & 0.067 & 0.038 & 0.080 \\
\hline & & $\mathrm{SE}$ & 0.074 & 0.040 & 0.086 & 0.072 & 0.038 & 0.084 \\
\hline & & $\mathrm{CP}(\%)$ & 92.4 & 92.4 & 94.0 & 91.8 & 93.6 & 94.8 \\
\hline & 0.5 & Bias & -0.016 & -0.002 & -0.005 & -0.015 & -0.003 & -0.003 \\
\hline & & $\mathrm{SD}$ & 0.080 & 0.045 & 0.092 & 0.076 & 0.041 & 0.087 \\
\hline & & $\mathrm{SE}$ & 0.085 & 0.046 & 0.100 & 0.083 & 0.044 & 0.096 \\
\hline & & $\mathrm{CP}(\%)$ & 93.6 & 92.4 & 95.0 & 92.4 & 93.4 & 95.6 \\
\hline & 0.75 & Bias & -0.006 & 0.003 & 0.003 & -0.005 & 0.003 & 0.003 \\
\hline & & $\mathrm{SD}$ & 0.064 & 0.036 & 0.074 & 0.061 & 0.034 & 0.072 \\
\hline & & SE & 0.070 & 0.037 & 0.081 & 0.069 & 0.038 & 0.081 \\
\hline & & $\mathrm{CP}(\%)$ & 94.2 & 95.2 & 94.4 & 94.6 & 94.8 & 96.2 \\
\hline \multirow[t]{12}{*}{$35 \%$} & 0.25 & Bias & -0.022 & 0.001 & -0.008 & -0.023 & 0.000 & -0.008 \\
\hline & & $\mathrm{SD}$ & 0.073 & 0.043 & 0.086 & 0.069 & 0.041 & 0.082 \\
\hline & & $\mathrm{SE}$ & 0.075 & 0.043 & 0.088 & 0.073 & 0.041 & 0.087 \\
\hline & & $\mathrm{CP}(\%)$ & 91.6 & 91.2 & 94.0 & 92.8 & 93.4 & 95.0 \\
\hline & 0.5 & Bias & -0.016 & -0.000 & -0.003 & -0.016 & -0.001 & -0.002 \\
\hline & & $\mathrm{SD}$ & 0.083 & 0.049 & 0.098 & 0.076 & 0.045 & 0.090 \\
\hline & & $\mathrm{SE}$ & 0.086 & 0.048 & 0.102 & 0.084 & 0.047 & 0.098 \\
\hline & & $\mathrm{CP}(\%)$ & 92.0 & 92.4 & 93.2 & 93.6 & 95.4 & 95.2 \\
\hline & 0.75 & Bias & -0.005 & 0.005 & 0.003 & -0.005 & 0.004 & 0.005 \\
\hline & & $\mathrm{SD}$ & 0.068 & 0.040 & 0.084 & 0.064 & 0.037 & 0.079 \\
\hline & & $\mathrm{SE}$ & 0.071 & 0.042 & 0.085 & 0.071 & 0.043 & 0.086 \\
\hline & & $\mathrm{CP}(\%)$ & 92.2 & 95.4 & 93.8 & 94.8 & 95.0 & 96.4 \\
\hline
\end{tabular}


表 2 异方差模型 $(\gamma=1)$ 下的随机模拟结果

\begin{tabular}{|c|c|c|c|c|c|c|c|c|}
\hline \multirow[b]{2}{*}{ 删失率 } & \multirow[b]{2}{*}{$\tau$} & & \multicolumn{3}{|c|}{ 最小化 $l_{1 j}$} & \multicolumn{3}{|c|}{ 最小化 $l_{j}$} \\
\hline & & & $\tilde{\beta}^{(0)}$ & $\tilde{\beta}^{(1)}$ & $\tilde{\beta}^{(2)}$ & $\hat{\beta}^{(0)}$ & $\hat{\beta}^{(1)}$ & $\hat{\beta}^{(2)}$ \\
\hline \multirow[t]{12}{*}{$20 \%$} & 0.25 & Bias & -0.021 & -0.001 & -0.041 & -0.022 & -0.001 & -0.040 \\
\hline & & SD & 0.096 & 0.072 & 0.132 & 0.092 & 0.069 & 0.127 \\
\hline & & $\mathrm{SE}$ & 0.099 & 0.077 & 0.142 & 0.097 & 0.074 & 0.138 \\
\hline & & $\mathrm{CP}(\%)$ & 93.0 & 93.8 & 93.6 & 93.2 & 94.2 & 92.6 \\
\hline & 0.5 & Bias & -0.021 & -0.003 & -0.027 & -0.014 & -0.004 & -0.034 \\
\hline & & SD & 0.105 & 0.081 & 0.139 & 0.093 & 0.071 & 0.126 \\
\hline & & $\mathrm{SE}$ & 0.109 & 0.085 & 0.156 & 0.105 & 0.080 & 0.148 \\
\hline & & $\mathrm{CP}(\%)$ & 93.8 & 95.0 & 96.4 & 94.6 & 95.4 & 97.0 \\
\hline & 0.75 & Bias & -0.014 & 0.005 & -0.008 & -0.006 & 0.004 & -0.016 \\
\hline & & $\mathrm{SD}$ & 0.087 & 0.061 & 0.114 & 0.078 & 0.054 & 0.098 \\
\hline & & $\mathrm{SE}$ & 0.092 & 0.064 & 0.122 & 0.090 & 0.062 & 0.118 \\
\hline & & $\mathrm{CP}(\%)$ & 94.2 & 94.2 & 94.4 & 96.2 & 95.0 & 97.0 \\
\hline \multirow[t]{12}{*}{$35 \%$} & 0.250 & Bias & -0.020 & -0.001 & -0.038 & -0.022 & -0.001 & -0.035 \\
\hline & & SD & 0.096 & 0.078 & 0.138 & 0.092 & 0.072 & 0.133 \\
\hline & & $\mathrm{SE}$ & 0.101 & 0.080 & 0.146 & 0.098 & 0.077 & 0.141 \\
\hline & & $\mathrm{CP}(\%)$ & 93.8 & 94.0 & 93.8 & 91.8 & 94.8 & 93.4 \\
\hline & 0.5 & Bias & -0.021 & 0.003 & -0.021 & -0.018 & 0.001 & -0.025 \\
\hline & & $\mathrm{SD}$ & 0.108 & 0.083 & 0.145 & 0.097 & 0.073 & 0.131 \\
\hline & & $\mathrm{SE}$ & 0.109 & 0.086 & 0.158 & 0.106 & 0.081 & 0.152 \\
\hline & & $\mathrm{CP}(\%)$ & 94.6 & 95.0 & 95.8 & 94.6 & 96.0 & 97.8 \\
\hline & 0.75 & Bias & -0.014 & 0.011 & -0.000 & -0.009 & 0.007 & -0.008 \\
\hline & & SD & 0.089 & 0.066 & 0.121 & 0.081 & 0.057 & 0.109 \\
\hline & & $\mathrm{SE}$ & 0.092 & 0.070 & 0.127 & 0.092 & 0.070 & 0.127 \\
\hline & & $\mathrm{CP}(\%)$ & 93.4 & 94.4 & 94.4 & 95.2 & 97.0 & 96.8 \\
\hline
\end{tabular}

设实验开始的时间为 100 , 而事件发生的时间服从 $[0,100]$ 上的均匀分布. 失效时间 $T^{0}$ 从如下模 型中生成,

$$
\log T^{0}=b_{1} Z_{1}+b_{2} Z_{2}+\left(1+\gamma Z_{2}\right) \varepsilon,
$$

其中 $\varepsilon$ 服从均匀分布 $U(0,1), Z_{1}$ 服从标准正态分布 $N(0,1)$, 而 $Z_{2}$ 服从成功概率为 0.5 的 Bernoulli 分布. 随机变量 $\varepsilon, Z_{1}$ 和 $Z_{2}$ 相互独立. 令 $\gamma=0$ 和 $\gamma=1$, 这分别对应同方差情形和异方差情形. 令系 数 $\left(b_{1}, b_{2}\right)$ 为 $(0.5,1)$. 记

$$
\boldsymbol{\beta}_{0}(\tau)=\left(\beta_{0}^{(0)}(\tau), \beta_{0}^{(1)}(\tau), \beta_{0}^{(2)}(\tau)\right)
$$

为对应协变量 $\left(1, Z_{1}, Z_{2}\right)$ 的分位数回归系数. 当 $\gamma=0$ 时,

$$
\left(\beta_{0}^{(0)}(\tau), \beta_{0}^{(1)}(\tau), \beta_{0}^{(2)}(\tau)\right)=\left(Q_{\varepsilon}(\tau), b_{1}, b_{2}\right),
$$

而当 $\gamma=1$ 时, $\beta_{0}^{(0)}(\tau)$ 和 $\beta_{0}^{(2)}(\tau)$ 的取值与 $\tau$ 有关, 但 $\beta_{0}^{(1)}(\tau)=b_{1}$ 取值与 $\tau$ 无关. 当 $Z_{1}<1$ 时, $C$ 服 从均匀分布 $U\left(0, c_{1}\right)$, 而当 $Z_{2}=1$ 时, $C$ 服从 $U\left(0, c_{2}\right)$, 其中 $c_{1}$ 和 $c_{2}$ 的取值使得删失率接近 $20 \%$ 或 
$35 \%$. 在每种情形下, 我们进行 500 次模拟, 样本量取为 $n=200$. 对于重抽样的情形, $\left\{\zeta_{i}, i=1, \ldots, n\right\}$ 服从均值为 1 的指数分布, $B$ 取值为 250 . 我们取等长的格子点 $\left\|\mathcal{S}_{L_{n}}\right\|=0.01$ 和 $M=10^{5}$.

我们报告了所提出统计量 $\tilde{\boldsymbol{\beta}}(\tau)$ 和 $\hat{\boldsymbol{\beta}}(\tau)$ 在 $\tau=0.25,0.5,0.75$ 时的经验偏差 (Bias)、经验标准差 (SD)、重抽样方法估计的平均标准差 (SE) 和 $95 \%$ Wald 型置信区间的经验覆盖率 $(\mathrm{CP})$. 模拟结果总 结在表 1 和 2 中.

从模拟结果我们可以看出, 所提出的估计量是无偏的, 重抽样方法估计的平均标准差和经验标准 差很接近, 置信区间具有合理的覆盖率. 从表 1 和 2 中的结果也可以得到, 对于长度偏差右删失数据 来讲, $\hat{\boldsymbol{\beta}}$ 的表现要比 $\tilde{\boldsymbol{\beta}}$ 更好一点, 这是因为 $\hat{\boldsymbol{\beta}}$ 的 $\mathrm{SE}$ 和 $\mathrm{SD}$ 要比 $\tilde{\boldsymbol{\beta}}$ 的更小. 这和我们预期是一致的, 因为 $\hat{\boldsymbol{\beta}}$ 利用了长度偏差数据的更多信息.

根据审稿人的建议, 我们也给出了同方差和异方差情形下, 忽略长度偏差数据时所得到的估计结 果, 它们分别在表 3 和 4 中给出. 从这两个表的随机模拟结果可以看出, 当不考虑长度偏差时, 所得到 的估计结果是很不好的, 尤其是 $95 \%$ 经验覆盖率有时会与名义上的 $95 \%$ 相差很远.

表 3 同方差模型 $(\gamma=0)$ 下忽略长度偏差而得到的随机模拟结果

\begin{tabular}{|c|c|c|c|c|c|c|c|c|}
\hline \multirow[b]{2}{*}{ 删失率 } & \multirow[b]{2}{*}{$\tau$} & & \multicolumn{3}{|c|}{ 最小化 $l_{1 j}$} & \multicolumn{3}{|c|}{ 最小化 $l_{j}$} \\
\hline & & & $\tilde{\beta}^{(0)}$ & $\tilde{\beta}^{(1)}$ & $\tilde{\beta}^{(2)}$ & $\hat{\beta}^{(0)}$ & $\hat{\beta}^{(1)}$ & $\hat{\beta}^{(2)}$ \\
\hline \multirow[t]{12}{*}{$20 \%$} & 0.25 & Bias & 0.110 & 0.012 & 0.017 & 0.076 & 0.006 & 0.005 \\
\hline & & $\mathrm{SD}$ & 0.070 & 0.037 & 0.078 & 0.074 & 0.041 & 0.087 \\
\hline & & SE & 0.065 & 0.033 & 0.074 & 0.071 & 0.038 & 0.083 \\
\hline & & $\mathrm{CP}(\%)$ & 58.4 & 89.0 & 91.6 & 79.8 & 91.6 & 93.8 \\
\hline & 0.5 & Bias & 0.027 & 0.002 & -0.005 & 0.080 & 0.011 & 0.014 \\
\hline & & $\mathrm{SD}$ & 0.072 & 0.039 & 0.084 & 0.049 & 0.025 & 0.056 \\
\hline & & $\mathrm{SE}$ & 0.073 & 0.039 & 0.085 & 0.049 & 0.025 & 0.055 \\
\hline & & $\mathrm{CP}(\%)$ & 92.8 & 93.2 & 93.8 & 58.2 & 89.4 & 91.8 \\
\hline & 0.75 & Bias & 0.008 & -0.003 & -0.005 & 0.040 & 0.001 & 0.001 \\
\hline & & $\mathrm{SD}$ & 0.061 & 0.034 & 0.072 & 0.075 & 0.040 & 0.087 \\
\hline & & $\mathrm{SE}$ & 0.062 & 0.035 & 0.073 & 0.076 & 0.041 & 0.087 \\
\hline & & $\mathrm{CP}(\%)$ & 92.6 & 92.6 & 93.4 & 90.6 & 95.0 & 94.0 \\
\hline \multirow[t]{12}{*}{$35 \%$} & 0.25 & Bias & 0.112 & 0.027 & 0.039 & 0.077 & 0.019 & 0.021 \\
\hline & & $\mathrm{SD}$ & 0.071 & 0.038 & 0.084 & 0.076 & 0.045 & 0.088 \\
\hline & & $\mathrm{SE}$ & 0.065 & 0.035 & 0.075 & 0.072 & 0.040 & 0.085 \\
\hline & & $\mathrm{CP}(\%)$ & 59.6 & 82.2 & 88.6 & 80.0 & 88.6 & 92.0 \\
\hline & 0.5 & Bias & 0.028 & 0.008 & 0.003 & 0.083 & 0.019 & 0.027 \\
\hline & & $\mathrm{SD}$ & 0.074 & 0.043 & 0.087 & 0.050 & 0.027 & 0.059 \\
\hline & & $\mathrm{SE}$ & 0.074 & 0.042 & 0.088 & 0.050 & 0.028 & 0.057 \\
\hline & & $\mathrm{CP}(\%)$ & 93.4 & 92.2 & 93.8 & 58.2 & 86.2 & 88.8 \\
\hline & 0.75 & Bias & 0.006 & -0.008 & -0.015 & 0.041 & 0.003 & 0.003 \\
\hline & & $\mathrm{SD}$ & 0.062 & 0.038 & 0.079 & 0.075 & 0.043 & 0.092 \\
\hline & & $\mathrm{SE}$ & 0.065 & 0.039 & 0.079 & 0.077 & 0.044 & 0.091 \\
\hline & & $\mathrm{CP}(\%)$ & 92.8 & 94.2 & 93.6 & 89.8 & 93.4 & 93.4 \\
\hline
\end{tabular}


表 4 异方差模型 $(\gamma=1)$ 下忽略长度偏差而得到的随机模拟结果

\begin{tabular}{|c|c|c|c|c|c|c|c|c|}
\hline \multirow[b]{2}{*}{ 删失率 } & \multirow[b]{2}{*}{$\tau$} & & \multicolumn{3}{|c|}{ 最小化 $l_{1 j}$} & \multicolumn{3}{|c|}{ 最小化 $l_{j}$} \\
\hline & & & $\tilde{\beta}^{(0)}$ & $\tilde{\beta}^{(1)}$ & $\tilde{\beta}^{(2)}$ & $\hat{\beta}^{(0)}$ & $\hat{\beta}^{(1)}$ & $\hat{\beta}^{(2)}$ \\
\hline \multirow{12}{*}{$20 \%$} & 0.25 & Bias & 0.107 & 0.040 & 0.396 & 0.073 & 0.029 & 0.370 \\
\hline & & $\mathrm{SD}$ & 0.068 & 0.054 & 0.101 & 0.074 & 0.063 & 0.129 \\
\hline & & $\mathrm{SE}$ & 0.060 & 0.047 & 0.089 & 0.070 & 0.060 & 0.121 \\
\hline & & $\mathrm{CP}(\%)$ & 54.2 & 80.6 & 2.0 & 77.4 & 90.0 & 16.0 \\
\hline & 0.5 & Bias & 0.021 & 0.008 & 0.200 & 0.084 & 0.027 & 0.238 \\
\hline & & $\mathrm{SD}$ & 0.075 & 0.066 & 0.139 & 0.049 & 0.036 & 0.069 \\
\hline & & $\mathrm{SE}$ & 0.073 & 0.064 & 0.133 & 0.046 & 0.037 & 0.068 \\
\hline & & $\mathrm{CP}(\%)$ & 93.0 & 92.8 & 65.2 & 49.4 & 86.0 & 6.0 \\
\hline & 0.75 & Bias & 0.006 & -0.027 & 0.012 & 0.036 & 0.002 & 0.181 \\
\hline & & $\mathrm{SD}$ & 0.059 & 0.053 & 0.107 & 0.072 & 0.060 & 0.121 \\
\hline & & SE & 0.062 & 0.055 & 0.108 & 0.071 & 0.061 & 0.121 \\
\hline & & $\mathrm{CP}(\%)$ & 93.2 & 89.2 & 94.0 & 90.8 & 93.4 & 68.4 \\
\hline \multirow[t]{12}{*}{$35 \%$} & 0.25 & Bias & 0.107 & 0.019 & 0.346 & 0.071 & 0.009 & 0.329 \\
\hline & & $\mathrm{SD}$ & 0.065 & 0.051 & 0.098 & 0.071 & 0.061 & 0.121 \\
\hline & & $\mathrm{SE}$ & 0.060 & 0.046 & 0.089 & 0.069 & 0.058 & 0.118 \\
\hline & & $\mathrm{CP}(\%)$ & 53.6 & 89.2 & 5.4 & 79.6 & 91.0 & 23.6 \\
\hline & 0.5 & Bias & 0.021 & 0.002 & 0.183 & 0.081 & 0.014 & 0.208 \\
\hline & & $\mathrm{SD}$ & 0.074 & 0.062 & 0.131 & 0.045 & 0.034 & 0.063 \\
\hline & & $\mathrm{SE}$ & 0.071 & 0.061 & 0.129 & 0.045 & 0.033 & 0.063 \\
\hline & & $\mathrm{CP}(\%)$ & 93.4 & 91.6 & 68.0 & 53.0 & 90.4 & 9.4 \\
\hline & 0.75 & Bias & 0.008 & -0.011 & 0.057 & 0.033 & 0.002 & 0.188 \\
\hline & & $\mathrm{SD}$ & 0.057 & 0.046 & 0.090 & 0.071 & 0.057 & 0.112 \\
\hline & & $\mathrm{SE}$ & 0.059 & 0.048 & 0.095 & 0.070 & 0.058 & 0.115 \\
\hline & & $\mathrm{CP}(\%)$ & 92.8 & 92.8 & 91.0 & 91.6 & 93.0 & 63.4 \\
\hline
\end{tabular}

\section{7 一个实际例子}

这里把提出的分位数回归模型的方法应用到美国 Channing House 数据上. Channing House 是美 国加利福尼亚州帕洛阿尔托市的一个退休中心. 美国 Channing House 数据集是一个左截断右删失数 据集 ${ }^{[37], ~}$ 它记录了 462 位退休人员, 包括 97 位男性和 365 位女性, 在 1964 年 1 月至 1975 年 7 月期 间进入退休中心的年龄和死亡年龄. 根据该记录, 总共有 46 位男性和 130 位女性在退休中心死亡. 有 趣的是, 文献 $[10,11]$ 发现仅包含进入年龄大于等于 65.5 岁的这些人组成的数据集是一个长度偏差右 删失数据集. 这个子集包含 448 个人, 因而只有 14 个人并未包括到该子集当中.

我们感兴趣的是研究在 Channing House 里男性与女性的生存差异. 为此, 用 $Z=1$ 表示男性个 体, $Z=0$ 表示女性个体. 这里在分析时, 我们用年作为时间单位. 考虑如下的回归模型:

$$
\log T^{0}=\alpha_{0}+\alpha_{1} Z+\varepsilon
$$


其中 $T^{0}$ 是无偏的生存时间.

我们利用估计方程 $(2.2)$ 和 $(2.4)$ 在 $\tau \in[0.15,0.55]$ 上拟合分位数回归模型. 用等距的格子点

$$
\left\|\mathcal{S}_{L_{n}}\right\|=0.02 .
$$

图 1 描述的是估计的分位数回归系数及相应的逐点 $95 \%$ 置信区间. 从两种方法可以得到类似的结果, 即女性常常比男性活的更长, 但是他们之间并没有显著的差异.

我们也用估计方程 (5.1) 和 (5.2) 做了模型拟合优度检验, 其中选取 $q(\cdot)$ 为个体性别的二次函数, 即 $[(Z-\mathrm{E}(Z)) / \operatorname{var}(Z)]^{2}$. 表 5 记录了 $p$ 值. 检验结果表明, 当我们把美国 Channing House 数据当作长 度偏差右删失数据来处理时, 分位数回归模型是合理的. 这个有趣的现象说明, 通过把该数据集看成 服从分位数回归模型 $(2.1)$ 的长度偏差右删失数据集, 我们可以预测或者估计其他量.

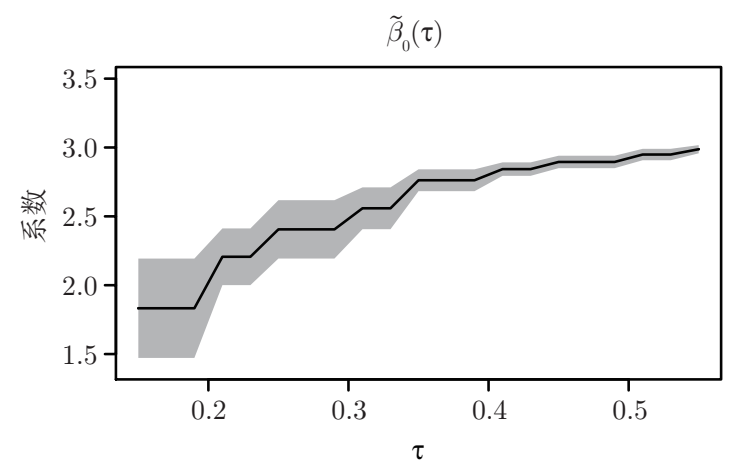

(a)

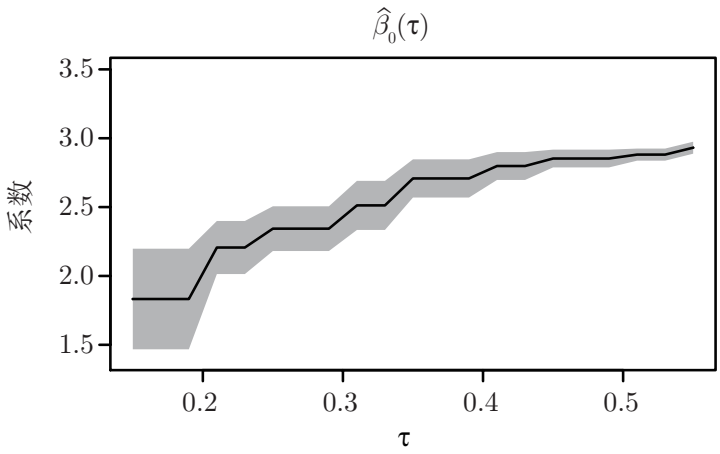

(c)

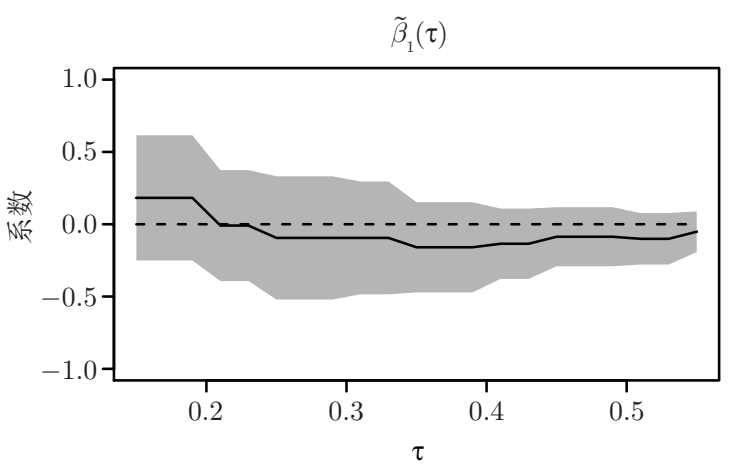

(b)

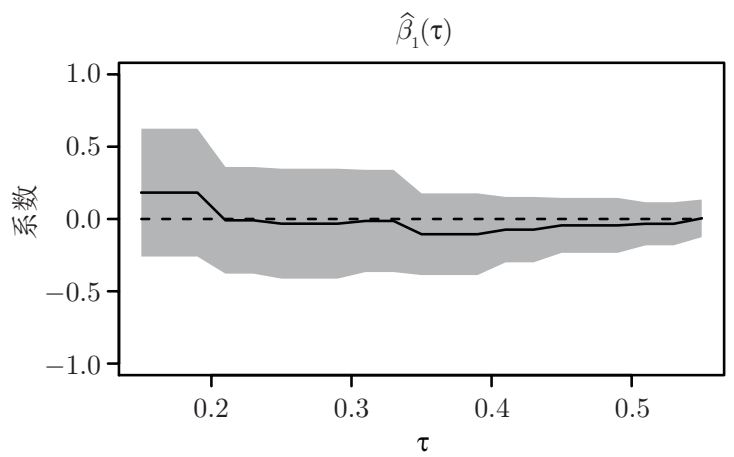

(d)

图 1 用两种方法得到的分位数回归系数

表 5 模型检验的 $p$ 值

\begin{tabular}{cccc}
\hline \multicolumn{2}{c}{$Q(\tau)$} & \multicolumn{2}{c}{$K(\tau)$} \\
$\tau$ & $p$ & $\tau$ & $p$ \\
\hline $0.15-0.25$ & 0.120 & $0.15-0.25$ & 0.132 \\
$0.25-0.35$ & 0.564 & $0.25-0.35$ & 0.592 \\
$0.35-0.45$ & 0.528 & $0.35-0.45$ & 0.872 \\
$0.45-0.55$ & $<0.0001$ & $0.45-0.55$ & 0.172 \\
\hline
\end{tabular}




\section{8 结束语}

在生存分析中, 分位数回归已经受到很大的重视, 因为在分析生存时间时, 分位数是比均值更加灵 活而且更加稳健的工具. 本文把文献 [24] 中提出的估计方程推广, 用来处理左截断右删失数据和长度 偏差右删失数据, 所得到的估计方程关于回归参数并不是光滑的. 我们通过极小化一系列 $L_{1}$ 类型的 凸函数来求解推广的估计方程, 并且建立了所提出估计量的大样本性质, 包括一致相合性和弱收玫性.

回归分位数在 $\tau$ 接近 1 时可能是不可识别的, 这是因为在后尾处缺失生存时间的信息. 因而, 我 们把注意力只放在估计小于或等于 $\tau_{U}$ 的那些分位点处. 一般而言, $\tau_{U}$ 应该满足一些可识别条件. 而 在实际例子分析中, $\tau_{U}$ 的选择是由我们感兴趣的问题所决定的.

模型 (2.1) 暗含着全局的线性假设, 这个假设有时是很强的, 因为回归分位数在某个点 $\tau$ 处的估 计需要在 $\tau$ 之前的所有回归分位数的估计. 如果这种线性条件只在某一特定的点 $\tau$ 处满足, 则可以沿 着这个思路推广文献 [25] 来分析长度偏差右删失数据. 也可以把所提出的方法推广到部分线性模型 来增加模型的适用性.

\section{参考文献}

1 Zelen M, Feinleib M. On the theory of screening for chronic diseases. Biometrika, 1969, 56: 601-614

2 Simon R. Length biased sampling in etiologic studies. Amer J Epidemiol, 1980, 111: 444-452

3 Winter B B, Folders A. A product-limit estimator for use with length-biased data. Canad J Statist, 1988, 16: 337-355

4 de Uña-Álvarez J, Otero-GirÁLdez M S, Alvarez-Llorente G. Estimation under length-bias and right-censoring: An application to unemployment duration analysis for married women. J Appl Stat, 2003, 30: 283-291

5 Asgharian M, Wolfson D. Asymptotic behavior of the unconditional NPMLE of the length-biased survivor function from right censored prevalent cohort data. Ann Statist, 2005, 33: 2109-2131

6 Shen Y, Ning J, Qin J. Analyzing length-biased data with semiparametric transformation and accelerated failure time models. J Amer Statist Assoc, 2009, 104: 1192-1202

7 de Uña-Álvarez J, Iglesias-Pérez M C. Nonparametric estimation of a conditional distribution from length-biased data. Ann Inst Statist Math, 2010, 62: 323-341

8 Qin J, Shen Y. Statistical methods for analyzing right-censored length-biased data under Cox model. Biometrics, 2010, 66: $382-392$

9 Huang C Y, Qin J. Composite partial likelihood estimation under length-biased sampling, with application to a prevalent cohort study of dementia. J Amer Statist Assoc, 2012, 107: 946-957

10 Chen X R, Zhou Y. Quantile regression for right-censored and length-biased data. Acta Math Appl Sin Engl Ser, 2012, 28: $443-462$

11 Zhang F P, Chen X R, Zhou Y. Proportional hazards model with varying coefficients for length-biased data. Lifetime Data Anal, 2014, 20: 132-157

12 Zhou X, Sun L, Ren H. Quantile estimation for left truncated and right censored data. Statist Sinica, 2000, 10: $1217-1230$

13 Huang C Y, Qin J. Nonparametric estimation for length-biased and right-censored data. Biometrika, 2011, 98: 177-186

14 Wang M, Brookmeyer R, Jewell N. Statistical models for prevalent cohort data. Biometrics, 1993, 49: 1-11

15 Huang C Y, Qin J. Semiparametric estimation for the additive hazards model with left-truncated and right-censored data. Biometrika, 2013, 100: 877-888

16 Wang M C. Hazards regression analysis for length-biased data. Biometrika, 1996, 83: 343-354

17 Shin Y. Length-bias correction in transformation models with supplementary data. Econometric Rev, 2009, 28: 658681

18 Tsai W Y. Pseudo-partial likelihood for proportional hazards models with biased-sampling data. Biometrika, 2009, 96: 601-615

19 Ning J, Qin J, Shen Y. Buckley-James-type estimator with right-censored and length-biased data. Biometrics, 2011, 67: $1369-1378$

20 Koenker R, Bassett J G. Regression quantiles. Econometrica, 1978, 46: 33-50 
21 Koenker. Quantile Regression. Cambridge: Cambridge University Press, 2005

22 Ying Z, Jung S, Wei L. Survival analysis with median regression models. J Amer Statist Assoc, 1995, 90: 178-184

23 Portnoy S. Censored regression quantiles. J Amer Statist Assoc, 2003, 98: 1001-1012

24 Peng L, Huang Y. Survival analysis with quantile regression models. J Amer Statist Assoc, 2008, 103: 637-649

25 Wang H, Wang L. Locally weighted censored quantile regression. J Amer Statist Assoc, 2009, 104: 1117-1128

26 Zhou W. A weighted quantile regression for randomly truncated data. Comput Statist Data Anal, 2011, 55: 554-566

27 Shen P S. A weighted quantile regression for left-truncated and right-censored data. J Stat Comput Simul, 2014, 84: 596-604

28 Wang H, Wang L. Quantile regression analysis of length-biased survival data. Statistics, 2014, 3: 31-47

29 Besag J E. Spatial interaction and the statistical analysis of lattice systems. J R Stat Soc Ser B Stat Methodol, 1974, 34: $192-236$

30 Lindsay B G. Composite likelihood methods. Contemp Math, 1988, 80: 221-239

31 Arnold B C, Strauss D. Bivariate distributions with exponential conditionals. J Amer Statist Assoc, 1988, 83: 522-527

32 Jin Z, Ying Z, Wei L J. A simple resampling method by perturbing the minimand. Biometrika, 2001, 88: 381-390

33 Andersen P K, Borgan O, Gill R D, et al. Statistical Models Based on Counting Processes. New York: Springer-Verlag, 1993

34 Zheng M, Zhao Z, Yu W. Quantile regression analysis of case-cohort data. J Multivariate Anal, 2013, 122: 20-34

35 Lin D Y, Wei L J, Ying Z. Checking the Cox model with cumulative sums of martingale-based residuals. Biometrika, 1993, 80: 557-572

36 Lin D Y, Wei L J, Yang I, et al. Semiparametric regression for the mean and rate functions of recurrent events. J R Stat Soc Ser B Stat Methodol, 2000, 62: 711-730

37 Zhao M, Jiang H, Liu X. A note on estimation of the mean residual life function with left-truncated and right-censored data. Statist Probab Lett, 2013, 83: 2332-2336

38 van der Vaart W. Weak Convergence and Empirical Processes with Applications to Statistics. New York: SpringerVerlag, 1996

\section{附录}

本附录给出 $\hat{\boldsymbol{\beta}}$ 的渐近结果.

\section{附录 A 一致相合性的证明}

$$
\begin{aligned}
\text { 定义 } \bar{\mu}(\boldsymbol{b}) & =\mathrm{E}\left[\boldsymbol{Z} R\left\{\exp \left(\boldsymbol{Z}^{\mathrm{T}} \boldsymbol{b}\right)\right\}\right], \\
\bar{\nu}_{n}(\boldsymbol{b}) & =n^{-1} \sum_{i=1}^{n} \boldsymbol{Z}_{i} R_{i}\left\{\exp \left(\boldsymbol{Z}_{i}^{\mathrm{T}} \boldsymbol{b}\right)\right\}-\bar{\mu}(\boldsymbol{b}), \quad \tilde{\nu}_{n}(\boldsymbol{b})=n^{-1} \sum_{i=1}^{n} \boldsymbol{Z}_{i} N_{i}\left\{\exp \left(\boldsymbol{Z}_{i}^{\mathrm{T}} \boldsymbol{b}\right)\right\}-\tilde{\mu}(\boldsymbol{b}) .
\end{aligned}
$$

为了简单起见, 我们记 $L_{n}=L$, 并假设 $\tau_{1}<\cdots<\tau_{L-1}$ 在 0 和 $\tau_{U}$ 之间等距. 记

$$
a_{n}=\left\|\mathcal{S}_{L}\right\|, \quad b_{n}=\frac{a_{n}}{1-\tau_{U}},
$$

则 $L=\tau_{U} / a_{n}$ 且对于 $j=1, \ldots, L$ 有 $0<H\left(\tau_{j}\right)-H\left(\tau_{j-1}\right) \leqslant b_{n}$.

引理 1 假设正则条件 $(\mathrm{C} 1)-(\mathrm{C} 4)$ 成立, 则 $\tilde{\mu}$ 在 $\mathcal{O}\left(\boldsymbol{\beta}_{0}, d_{0}\right)$ 上是可逆的.

证明 与文献 $[24$, 附录 $\mathrm{A}]$ 的证明类似. 所以, 我们在这里省略细节.

引理 2 假设正则条件 $(\mathrm{C} 1)-(\mathrm{C} 5)$ 成立, 则

$$
\sup _{0<\tau<\tau_{U}}\left\|\tilde{\mu}\{\hat{\boldsymbol{\beta}}(\tau)\}-\tilde{\mu}\left\{\boldsymbol{\beta}_{0}(\tau)\right\}\right\| \rightarrow_{p} 0 .
$$


证明 经过一些简单的代数计算, 我们有

$$
\frac{1}{n} \sum_{i=1}^{n} \boldsymbol{Z}_{i} N_{i}\left(\exp \left\{\boldsymbol{Z}_{i}^{\mathrm{T}} \hat{\boldsymbol{\beta}}\left(\tau_{j}\right)\right\}\right)-\mathrm{E}\left[\boldsymbol{Z} N\left(\exp \left\{\boldsymbol{Z}^{\mathrm{T}} \boldsymbol{\beta}_{0}\left(\tau_{j}\right)\right\}\right)\right]=\tilde{\nu}_{n}\left\{\hat{\boldsymbol{\beta}}\left(\tau_{j}\right)\right\}+\tilde{\mu}\left\{\hat{\boldsymbol{\beta}}\left(\tau_{j}\right)\right\}-\tilde{\mu}\left\{\boldsymbol{\beta}_{0}\left(\tau_{j}\right)\right\}
$$

和

$$
\begin{aligned}
\frac{1}{n} & \sum_{i=1}^{n} \int_{0}^{\tau_{j}} \boldsymbol{Z}_{i} R_{i}\left(\exp \left\{\boldsymbol{Z}_{i}^{\mathrm{T}} \hat{\boldsymbol{\beta}}(u)\right\}\right) d H(u)-\mathrm{E}\left[\int_{0}^{\tau_{j}} \boldsymbol{Z} R\left(\exp \left\{\boldsymbol{Z}^{\mathrm{T}} \boldsymbol{\beta}_{0}(u)\right\}\right) d H(u)\right] \\
& =\int_{0}^{\tau_{j}} \bar{\nu}_{n}\{\hat{\boldsymbol{\beta}}(u)\} d H(u)+\int_{0}^{\tau_{j}}\left[\bar{\mu}\{\hat{\boldsymbol{\beta}}(u)\}-\bar{\mu}\left\{\boldsymbol{\beta}_{0}(u)\right\}\right] d H(u) .
\end{aligned}
$$

注意到, 对于 $j=1, \ldots, L$,

$$
\begin{aligned}
& \mathrm{E}\left[\boldsymbol{Z} N\left(\exp \left\{\boldsymbol{Z}^{\mathrm{T}} \boldsymbol{\beta}_{0}\left(\tau_{j}\right)\right\}\right)\right]=\mathrm{E}\left[\int_{0}^{\tau_{j}} \boldsymbol{Z} R\left(\exp \left\{\boldsymbol{Z}^{\mathrm{T}} \boldsymbol{\beta}_{0}(u)\right\}\right) d H(u)\right] \\
& \frac{1}{n} \sum_{i=1}^{n} \boldsymbol{Z}_{i} N_{i}\left(\exp \left\{\boldsymbol{Z}_{i}^{\mathrm{T}} \hat{\boldsymbol{\beta}}\left(\tau_{j}\right)\right\}\right)=\frac{1}{n} \sum_{i=1}^{n} \int_{0}^{\tau_{j}} \boldsymbol{Z}_{i} R_{i}\left(\exp \left\{\boldsymbol{Z}_{i}^{\mathrm{T}} \hat{\boldsymbol{\beta}}(u)\right\}\right) d H(u)+\varsigma_{j n},
\end{aligned}
$$

其中

$$
\max _{1 \leqslant j \leqslant L}\left\|\varsigma_{j n}\right\| \leqslant \frac{\sup _{i}\left\|\boldsymbol{Z}_{i}\right\|}{n}
$$

从以上四个方程中可以得到

$$
\begin{aligned}
\tilde{\mu}\left\{\hat{\boldsymbol{\beta}}\left(\tau_{j}\right)\right\}-\tilde{\mu}\left\{\boldsymbol{\beta}_{0}\left(\tau_{j}\right)\right\}= & -\tilde{\nu}_{n}\left\{\hat{\boldsymbol{\beta}}\left(\tau_{j}\right)\right\}+\int_{0}^{\tau_{j}} \bar{\nu}_{n}\{\hat{\boldsymbol{\beta}}(u)\} d H(u) \\
& +\sum_{k=1}^{j} \int_{\tau_{k-1}}^{\tau_{k}}\left[\bar{\mu}\{\hat{\boldsymbol{\beta}}(u)\}-\bar{\mu}\left\{\boldsymbol{\beta}_{0}(u)\right\}\right] d H(u)+\varsigma_{j n} .
\end{aligned}
$$

方程 (A.1) 是得到该定理的关键. 根据在第 3 节中给出的正则条件, 我们有如下有用的结果:

(1) 定义

$$
M_{1 n}=\sup _{j}\left\|-\tilde{\nu}_{n}\left\{\hat{\boldsymbol{\beta}}\left(\tau_{j}\right)\right\}+\int_{0}^{\tau_{j}} \bar{\nu}_{n}\{\hat{\boldsymbol{\beta}}(u)\} d H(u)\right\|
$$

由于

$$
\begin{aligned}
& \mathcal{G}_{1}=\left\{\boldsymbol{Z}_{i} I\left\{Y_{i} \leqslant \exp \left(\boldsymbol{Z}_{i}^{\mathrm{T}} \boldsymbol{b}\right)\right\} \delta_{i}: \boldsymbol{b} \in \mathbb{R}^{p}\right\}, \\
& \mathcal{G}_{2}=\left\{\frac{1}{2} \boldsymbol{Z}_{i}\left[I\left\{A_{i} \leqslant \exp \left(\boldsymbol{Z}_{i}^{\mathrm{T}} \boldsymbol{b}\right) \leqslant Y_{i}\right\}+I\left\{\tilde{V}_{i} \leqslant \exp \left(\boldsymbol{Z}_{i}^{\mathrm{T}} \boldsymbol{b}\right) \leqslant Y_{i}\right\}\right]: \boldsymbol{b} \in \mathbb{R}^{p}\right\}
\end{aligned}
$$

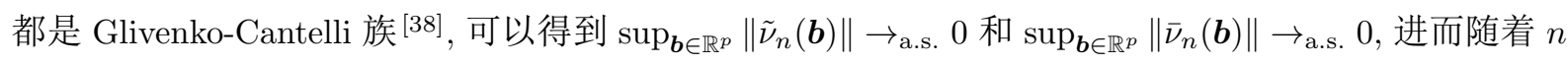
趋于无穷, $M_{1 n} \rightarrow_{\text {a.s. }} 0$.

(2) 存在 $M_{2}>0$ 使得

$$
\sup _{i}\left\|\boldsymbol{Z}_{i}\right\| \leqslant M_{2} .
$$

(3) 由 $\tilde{\mu}$ 的 Lipschitz 性质可以得到, 存在常数 $M_{3}>0$, 对于任意的 $\tau, \tau^{\prime} \in\left(0, \tau_{U}\right]$,

$$
\left\|\tilde{\mu}\left\{\boldsymbol{\beta}_{0}(\tau)\right\}-\tilde{\mu}\left\{\boldsymbol{\beta}_{0}\left(\tau^{\prime}\right)\right\}\right\| \leqslant M_{3}\left|\tau-\tau^{\prime}\right| .
$$


(4) 存在 $M_{4}>0$ 使得对于任意的 $\boldsymbol{x} \in \mathbb{R}^{p}$ 和 $\boldsymbol{b} \in \mathcal{B}\left(\boldsymbol{\beta}_{0}, d_{0}\right)$ 有

$$
\left\|B(\boldsymbol{b})^{-1} J(\boldsymbol{b}) \boldsymbol{x}\right\| \leqslant C_{4}\|\boldsymbol{x}\| .
$$

定义序列 $\left\{\varepsilon_{l n}\right\}_{l=0}^{L-1}$, 其中 $\varepsilon_{0 n}=M_{3} a_{n}$, 对于 $l=1, \ldots, L-1$, 有

$$
\varepsilon_{l n}=M_{1 n}+\left(\sum_{k=0}^{l-1} \varepsilon_{k n}\right) M_{4} b_{n}+M_{2} n^{-1}+M_{3} a_{n} .
$$

之后我们用数学归纳法来证明, 对于 $l=0, \ldots, L-1$, 有

$$
\sup _{\tau_{l} \leqslant \tau<\tau_{l+1}}\left\|\tilde{\mu}\{\hat{\boldsymbol{\beta}}(\tau)\}-\tilde{\mu}\left\{\boldsymbol{\beta}_{0}(\tau)\right\}\right\| \leqslant \varepsilon_{l n}
$$

首先, 根据 $\hat{\boldsymbol{\beta}}(\tau)$ 的定义, 我们有

$$
\sup _{\tau_{0} \leqslant \tau<\tau_{1}}\left\|\tilde{\mu}\{\hat{\boldsymbol{\beta}}(\tau)\}-\tilde{\mu}\left\{\boldsymbol{\beta}_{0}(\tau)\right\}\right\|=\sup _{\tau_{0} \leqslant \tau<\tau_{1}}\left\|\tilde{\mu}\left\{\boldsymbol{\beta}_{0}(\tau)\right\}\right\| \leqslant M_{3} a_{n}=\varepsilon_{0 n} .
$$

其次, 假设对于 $\forall k \in\{0, \ldots, j-1\}$, 有

$$
\sup _{\tau_{k} \leqslant \tau<\tau_{k+1}} \| \tilde{\mu}\left\{\hat{\boldsymbol{\beta}}(\tau)-\tilde{\mu}\left\{\boldsymbol{\beta}_{0}(\tau)\right\} \| \leqslant \varepsilon_{k n}\right.
$$

这意味着

$$
\sup _{\tau_{k} \leqslant \tau<\tau_{k+1}}\left\|\bar{\mu}\{\hat{\boldsymbol{\beta}}(\tau)\}-\bar{\mu}\left\{\boldsymbol{\beta}_{0}(\tau)\right\}\right\| \leqslant M_{4} \varepsilon_{k n} .
$$

接下来, 我们证明

$$
\sup _{\tau_{j} \leqslant \tau<\tau_{j+1}}\left\|\tilde{\mu}\{\hat{\boldsymbol{\beta}}(\tau)\}-\tilde{\mu}\left\{\boldsymbol{\beta}_{0}(\tau)\right\}\right\| \leqslant \varepsilon_{j n} .
$$

由 (A.1), 根据前面的方程和一些简单的代数计算可以得到

$$
\left\|\tilde{\mu}\left\{\hat{\boldsymbol{\beta}}\left(\tau_{j}\right)\right\}-\tilde{\mu}\left\{\boldsymbol{\beta}_{0}\left(\tau_{j}\right)\right\}\right\| \leqslant M_{1 n}+\left(\sum_{k=0}^{j-1} \varepsilon_{k n}\right) M_{4} b_{n}+M_{2} n^{-1} .
$$

利用三角不等式,

$$
\begin{aligned}
\sup _{\tau_{j} \leqslant \tau<\tau_{j+1}}\left\|\tilde{\mu}\{\hat{\boldsymbol{\beta}}(\tau)\}-\tilde{\mu}\left\{\boldsymbol{\beta}_{0}(\tau)\right\}\right\| & =\sup _{\tau_{j} \leqslant \tau<\tau_{j+1}}\left\|\tilde{\mu}\left\{\hat{\boldsymbol{\beta}}\left(\tau_{j}\right)\right\}-\tilde{\mu}\left\{\boldsymbol{\beta}_{0}(\tau)\right\}\right\| \\
& \leqslant\left\|\tilde{\mu}\left\{\hat{\boldsymbol{\beta}}\left(\tau_{j}\right)\right\}-\tilde{\mu}\left\{\boldsymbol{\beta}_{0}\left(\tau_{j}\right)\right\}\right\|+\sup _{\tau_{j} \leqslant \tau<\tau_{j+1}}\left\|\tilde{\mu}\left\{\boldsymbol{\beta}_{0}\left(\tau_{j}\right)\right\}-\tilde{\mu}\left\{\boldsymbol{\beta}_{0}(\tau)\right\}\right\| \\
& \leqslant M_{1 n}+\left(\sum_{k=0}^{j-1} \varepsilon_{k n}\right) M_{4} b_{n}+M_{2} n^{-1}+M_{3} a_{n}=\varepsilon_{j n} .
\end{aligned}
$$

从 $\varepsilon_{j n}$ 的定义, 我们有

$$
\varepsilon_{j n}=\left(1+M_{4} b_{n}\right)^{j-1}\left(M_{1 n}+\varepsilon_{0 n} M_{4} b_{n}+M_{2} n^{-1}+M_{3} a_{n}\right) .
$$


在给定 $\lim _{n \rightarrow \infty} a_{n}=0$ 和 $L=\tau_{U} / a_{n}$ 时, 我们有

$$
\lim _{n \rightarrow \infty}\left(1+M_{4} b_{n}\right)^{L-1}=\exp \left\{\frac{M_{4} \tau_{U}}{1-\tau_{U}}\right\} .
$$

然后很容易得到, 对于 $j=0, \ldots, L$, 有 $\varepsilon_{j n} \rightarrow 0$, 进而,

$$
\sup _{0<\tau<\tau_{U}}\left\|\tilde{\mu}\{\hat{\boldsymbol{\beta}}(\tau)\}-\tilde{\mu}\left\{\boldsymbol{\beta}_{0}(\tau)\right\}\right\| \rightarrow_{p} 0 .
$$

引理证毕.

一致相合性的证明 结合引理 2、反函数定理和连续映射定理, 随着 $n \rightarrow \infty$, 对于 $\forall \nu \in\left(0, \tau_{U}\right)$, 我们有

$$
\sup _{\tau \in\left[\nu, \tau_{U}\right]}\left\|\hat{\boldsymbol{\beta}}(\tau)-\boldsymbol{\beta}_{0}(\tau)\right\| \rightarrow_{p} 0
$$

证毕.

\section{附录 B 弱收敛性的证明}

引理 3 对任何满足 $\sup _{\tau \in\left(0, \tau_{U}\right]}\left\|\tilde{\mu}\left\{\hat{\boldsymbol{\beta}}_{n}(\tau)\right\}-\tilde{\mu}\left\{\boldsymbol{\beta}_{0}(\tau)\right\}\right\| \rightarrow_{p} 0$ 的序列 $\left\{\hat{\boldsymbol{\beta}}_{n}(\tau), \tau \in\left(0, \tau_{U}\right]\right\}_{n=1}^{\infty}$, 我 们有

$$
\begin{aligned}
& \sup _{\tau \in\left(0, \tau_{U}\right]} n^{1 / 2}\left\|\tilde{\nu}_{n}\left\{\hat{\boldsymbol{\beta}}_{n}(\tau)\right\}-\tilde{\nu}_{n}\left\{\boldsymbol{\beta}_{0}(\tau)\right\}\right\| \rightarrow_{p} 0 \\
& \sup _{\tau \in\left(0, \tau_{U}\right]} n^{1 / 2}\left\|\bar{\nu}_{n}\left\{\hat{\boldsymbol{\beta}}_{n}(\tau)\right\}-\bar{\nu}_{n}\left\{\boldsymbol{\beta}_{0}(\tau)\right\}\right\| \rightarrow_{p} 0 .
\end{aligned}
$$

引理 3 的证明可以由文献 [24, 引理 B.1] 和 [34, 引理 A.8] 得到.

引理 4 在正则条件 (C1) 和 (C2) 成立时, 对于 $\tau \in\left(0, \tau_{U}\right]$, 随着 $n \rightarrow \infty, n^{1 / 2} \boldsymbol{S}_{n}\left(\boldsymbol{\beta}_{0}, \tau\right)$ 弱收敛 到一个胎紧的 Gauss 过程 $G(\tau)$, 该 Gauss 过程的均值为 0 , 协方差函数为 $\Sigma(s, t)$, 其中

$$
\Sigma(s, t)=\mathrm{E}\left\{\iota_{j}(s) \iota_{j}(t)^{\mathrm{T}}\right\}, \quad \iota_{i}(\tau)=\boldsymbol{Z}_{i}\left[N_{i}\left(\exp \left\{\boldsymbol{Z}_{i}^{\mathrm{T}} \boldsymbol{\beta}_{0}(\tau)\right\}\right)-\int_{0}^{\tau} R_{i}\left(\exp \left\{\boldsymbol{Z}_{i}^{\mathrm{T}} \boldsymbol{\beta}_{0}(u)\right\}\right) d H(u)\right] .
$$

证明 因为

$$
\left\{\boldsymbol{Z}_{i}\left[N_{i}\left(\exp \left\{\boldsymbol{Z}_{i}^{\mathrm{T}} \boldsymbol{\beta}_{0}(\tau)\right\}\right)-\int_{0}^{\tau} R_{i}\left(\exp \left\{\boldsymbol{Z}_{i}^{\mathrm{T}} \boldsymbol{\beta}_{0}(u)\right\}\right) d H(u)\right], \tau \in\left(0, \tau_{U}\right]\right\}
$$

是一个 Donsker 族 ${ }^{[38]}$, 根据 Donsker 定理, 可以立即得到该引理的结论.

弱收玫性的证明 从定理 1 的证明和引理 3 , 我们可以得到

$$
\begin{aligned}
& \sup _{\tau \in\left(0, \tau_{U}\right]} n^{1 / 2}\left\|\tilde{\nu}_{n}(\hat{\boldsymbol{\beta}}(\tau))-\tilde{\nu}_{n}\left(\boldsymbol{\beta}_{0}(\tau)\right)\right\| \rightarrow_{p} 0, \\
& \sup _{\tau \in\left(0, \tau_{U}\right]} n^{1 / 2}\left\|\bar{\nu}_{n}(\hat{\boldsymbol{\beta}}(\tau))-\bar{\nu}_{n}\left(\boldsymbol{\beta}_{0}(\tau)\right)\right\| \rightarrow_{p} 0 .
\end{aligned}
$$


记 $o_{I}(1)$ 为在 $\tau \in I$ 上依概率一致收玫于 0 的项. 根据 $\boldsymbol{S}_{n}(\hat{\boldsymbol{\beta}}, \tau)$ 的定义,

$$
\sup _{\tau \in\left[\tau_{j}, \tau_{j+1}\right)} n^{1 / 2}\left\|\boldsymbol{S}_{n}(\hat{\boldsymbol{\beta}}, \tau)-\boldsymbol{S}_{n}\left(\hat{\boldsymbol{\beta}}, \tau_{j}\right)\right\| \leqslant n^{1 / 2} 2 M_{2}\left\{H\left(\tau_{j+1}\right)-H\left(\tau_{j}\right)\right\} \leqslant \frac{n^{1 / 2} 2 M_{2} a_{n}}{1-\tau_{U}} .
$$

因为 $n^{1 / 2} a_{n} \rightarrow 0$, 我们有 $n^{1 / 2} \boldsymbol{S}_{n}(\hat{\boldsymbol{\beta}}, \tau)=o_{\left(0, \tau_{U}\right]}(1)$, a.s., 则由 (B.1)、(B.2) 和在 $\tau \in\left(0, \tau_{U}\right]$ 上, $\tilde{\mu}\{\hat{\boldsymbol{\beta}}(\tau)\}$ 一致收玫到 $\tilde{\mu}\left\{\boldsymbol{\beta}_{0}(\tau)\right\}$, 可以得到

$$
\begin{aligned}
-n^{1 / 2} \boldsymbol{S}_{n}\left(\boldsymbol{\beta}_{0}, \tau\right)= & n^{1 / 2}\left[\tilde{\mu}\{\hat{\boldsymbol{\beta}}(\tau)\}-\tilde{\mu}\left\{\boldsymbol{\beta}_{0}(\tau)\right\}\right]-\int_{0}^{\tau} n^{1 / 2}\left[\bar{\mu}\{\hat{\boldsymbol{\beta}}(u)\}-\bar{\mu}\left\{\boldsymbol{\beta}_{0}(u)\right\}\right] d H(u)+o_{\left(0, \tau_{U}\right]}(1) \\
= & n^{1 / 2}\left[\tilde{\mu}\{\hat{\boldsymbol{\beta}}(\tau)\}-\tilde{\mu}\left\{\boldsymbol{\beta}_{0}(\tau)\right\}\right]-\int_{0}^{\tau}\left[J\left\{\boldsymbol{\beta}_{0}(u)\right\} B\left\{\boldsymbol{\beta}_{0}(u)\right\}^{-1}\right] n^{1 / 2}[\tilde{\mu}\{\hat{\boldsymbol{\beta}}(u)\} \\
& \left.-\tilde{\mu}\left\{\boldsymbol{\beta}_{0}(u)\right\}\right] d H(u)+o_{\left(0, \tau_{U}\right]}(1) .
\end{aligned}
$$

上面的这个方程对 $n^{1 / 2}\left[\tilde{\mu}\{\hat{\boldsymbol{\beta}}(\tau)\}-\tilde{\mu}\left\{\boldsymbol{\beta}_{0}(\tau)\right\}\right]$ 而言是一个随机微分方程. 运用乘积积分理论 ${ }^{[33]}$, 我们 可以得到

$$
n^{1 / 2}\left[\tilde{\mu}\{\hat{\boldsymbol{\beta}}(\tau)\}-\tilde{\mu}\left\{\boldsymbol{\beta}_{0}(\tau)\right\}\right]=\phi\left\{-n^{1 / 2} S_{n}\left(\boldsymbol{\beta}_{0}, \tau\right)\right\}+o_{\left(0, \tau_{U}\right]}(1),
$$

其中 $\phi$ 是一个从 $\mathcal{F}$ 到 $\mathcal{F}$ 的映射, 使得对于 $g \in \mathcal{F}$,

$$
\phi(g)(t)=\int_{0}^{t} \mathcal{I}(s, t) d g(s),
$$

这里

$$
\begin{aligned}
& \mathcal{I}(s, t)=\prod_{u \in(s, t]}\left[I_{p}+J\left\{\boldsymbol{\beta}_{0}(u)\right\} B\left\{\boldsymbol{\beta}_{0}(u)\right\}^{-1}\right] d H(u), \\
& \mathcal{F}=\left\{g:\left[0, \tau_{U}\right] \rightarrow \mathbb{R}^{p}, g \text { 左连续且具有右极限, } g(0)=0\right\} .
\end{aligned}
$$

因为 $\phi$ 是一个线性算子, 因而对于 $\tau \in\left(0, \tau_{U}\right]$, 随机过程 $\left\{n^{1 / 2}\left[\tilde{\mu}\{\hat{\boldsymbol{\beta}}(\tau)\}-\tilde{\mu}\left\{\boldsymbol{\beta}_{0}(\tau)\right\}\right]: \tau \in\left(0, \tau_{U}\right]\right\}$ 弱收 玫到一个零均值的 Gauss 过程 $\phi\{G(\tau)\}$. 最后, 利用反函数定理和连续映射定理, 我们可以得到, 随着 $n \rightarrow \infty$, 过程 $\left\{n^{1 / 2}\left[\hat{\boldsymbol{\beta}}(\tau)-\boldsymbol{\beta}_{0}(\tau)\right]: \tau \in\left[\nu, \tau_{U}\right]\right\}$ 弱收玫到 $B\left\{\boldsymbol{\beta}_{0}(\tau)\right\}^{-1} \phi\{G(\tau)\}$, 这也是一个 Gauss 过 程. 证毕.

\section{附录 C 重抽样技巧的论证}

定义

$$
\boldsymbol{S}_{n}^{*}(\boldsymbol{\beta}, \tau)=n^{-1} \sum_{i=1}^{n} \zeta_{i} \boldsymbol{Z}_{i}\left[N_{i}\left(\exp \left\{\boldsymbol{Z}_{i}^{\mathrm{T}} \boldsymbol{\beta}(\tau)\right\}\right)-\int_{0}^{\tau} R_{i}\left(\exp \left\{\boldsymbol{Z}_{i}^{\mathrm{T}} \boldsymbol{\beta}(u)\right\}\right) d H(u)\right] .
$$

给定 $\mathrm{E}\left(\zeta_{i}\right)=1$, 类似于附录 B 中给出的论证, 我们可以得到 $n^{1 / 2} \boldsymbol{S}_{n}^{*}\left(\hat{\boldsymbol{\beta}}^{*}, \tau\right)=o_{\left(0, \tau_{U}\right]}(1)$, a.s., 且

$$
n^{1 / 2}\left\{\hat{\boldsymbol{\beta}}^{*}(\tau)-\hat{\boldsymbol{\beta}}(\tau)\right\}=B\left\{\boldsymbol{\beta}_{0}(\tau)\right\}^{-1} \phi\left\{-n^{1 / 2} \boldsymbol{S}_{n}^{*}(\hat{\boldsymbol{\beta}}, \tau)\right\}+o_{\left(0, \tau_{U}\right]}(1) .
$$

1998 
因为 $n^{1 / 2} \boldsymbol{S}_{n}(\hat{\boldsymbol{\beta}}, \tau)=o_{\left(0, \tau_{U}\right]}(1)$, a.s., 类似地可以证明

$$
-n^{1 / 2} \boldsymbol{S}_{n}^{*}(\hat{\boldsymbol{\beta}}, \tau)=n^{1 / 2}\left\{\boldsymbol{S}_{n}(\hat{\boldsymbol{\beta}}, \tau)-\boldsymbol{S}_{n}^{*}(\hat{\boldsymbol{\beta}}, \tau)\right\}+o_{\left(0, \tau_{U}\right]}(1)=n^{-1 / 2} \sum_{j=1}^{n}\left(1-\zeta_{j}\right) \hat{\iota}_{j}(\tau)+o_{\left(0, \tau_{U}\right]}(1),
$$

其中

$$
\hat{\iota}_{j}(\tau)=\boldsymbol{Z}_{j}\left[N_{j}\left(\exp \left\{\boldsymbol{Z}_{j}^{\mathrm{T}} \hat{\boldsymbol{\beta}}(\tau)\right\}\right)-\int_{0}^{\tau} R_{j}\left(\exp \left\{\boldsymbol{Z}_{j}^{\mathrm{T}} \hat{\boldsymbol{\beta}}(u)\right\}\right) d H(u)\right] .
$$

利用 $\operatorname{var}\left(\zeta_{j}\right)=1$, 对 $s, t \in\left[\nu, \tau_{U}\right]$, 我们有

$$
\mathrm{E}\left[n \boldsymbol{S}_{n}^{*}(\hat{\boldsymbol{\beta}}, s) \boldsymbol{S}_{n}^{*}(\hat{\boldsymbol{\beta}}, t)^{\mathrm{T}} \mid\left\{A_{i}, Y_{i}, \delta_{i}, \boldsymbol{Z}_{i}\right\}_{i=1}^{n}\right]=n^{-1} \sum_{j=1}^{n} \hat{\iota}_{j}(s) \hat{\iota}_{j}(t)^{\mathrm{T}} \rightarrow_{p} \Sigma(s, t) .
$$

根据文献 [36], 给定数据 $\left\{A_{i}, Y_{i}, \delta_{i}, \boldsymbol{Z}_{i}\right\}_{i=1}^{n}$ 的几乎所有实现值, $-n^{1 / 2} \boldsymbol{S}_{n}^{*}(\hat{\boldsymbol{\beta}}, \tau)$ 的分布与 $-n^{1 / 2} \boldsymbol{S}_{n}\left(\boldsymbol{\beta}_{0}, \tau\right)$ 的分布收玫到相同的极限. 可以从 (C.1) 和 (C.2) 中得到, 对于 $\tau \in\left[\nu, \tau_{U}\right]$, 在给定观测数据的条件下, $n^{1 / 2}\left\{\hat{\boldsymbol{\beta}}^{*}(\tau)-\hat{\boldsymbol{\beta}}(\tau)\right\}$ 的条件分布和 $n^{1 / 2}\left\{\hat{\boldsymbol{\beta}}(\tau)-\boldsymbol{\beta}_{0}(\tau)\right\}$ 的无条件分布是渐近等价的.

\section{附录 D 模型检验的论证}

根据 $K(\cdot)$ 的定义, 我们有

$$
\begin{aligned}
K(\tau) & =n^{-1 / 2} \sum_{i=1}^{n} q\left(\boldsymbol{Z}_{i}\right) M_{i}\left(\tau ; \boldsymbol{\beta}_{0}\right)+n^{-1 / 2} \sum_{i=1}^{n} q\left(\boldsymbol{Z}_{i}\right)\left\{M_{i}(\tau ; \hat{\boldsymbol{\beta}})-M_{i}\left(\tau ; \boldsymbol{\beta}_{0}\right)\right\} \\
& =n^{-1 / 2} \sum_{i=1}^{n} q\left(\boldsymbol{Z}_{i}\right) M_{i}\left(\tau ; \boldsymbol{\beta}_{0}\right)+\psi\left(n^{1 / 2}\left\{\hat{\boldsymbol{\beta}}(\tau)-\boldsymbol{\beta}_{0}(\tau)\right\}\right)+o_{\left(\nu, \tau_{U}\right]}(1),
\end{aligned}
$$

其中 $\psi$ 是一个从 $\mathcal{F}$ 到 $\tilde{\mathcal{F}}$ 的线性映射. $\psi$ 的定义如下:

$$
\begin{aligned}
\psi(g)= & \mathrm{E}\left[q(\boldsymbol{Z}) \tilde{f}\left(\exp \left\{\boldsymbol{Z}^{\mathrm{T}} \boldsymbol{\beta}_{0}(\tau)\right\} \mid \boldsymbol{Z}\right) \exp \left\{\boldsymbol{Z}^{\mathrm{T}} \boldsymbol{\beta}_{0}(\tau)\right\} \boldsymbol{Z}^{\mathrm{T}}\right] g(\tau) \\
& -\int_{0}^{\tau} \mathrm{E}\left[q(\boldsymbol{Z}) \bar{f}\left(\exp \left\{\boldsymbol{Z}^{\mathrm{T}} \boldsymbol{\beta}_{0}(\tau)\right\} \mid \boldsymbol{Z}\right) \exp \left\{\boldsymbol{Z}^{\mathrm{T}} \boldsymbol{\beta}_{0}(u)\right\} \boldsymbol{Z}^{\mathrm{T}}\right] g(u) d H(u),
\end{aligned}
$$

其中 $g \in \mathcal{F}$ 且 $\tilde{\mathcal{F}}=\left\{h:\left[0, \tau_{U}\right] \rightarrow \mathbb{R}, h\right.$ 是 cadlag 的, $\left.h(0)=0\right\}$. 从附录 B 可以得到 $n^{1 / 2}\left\{\hat{\boldsymbol{\beta}}(\tau)-\boldsymbol{\beta}_{0}(\tau)\right\}$ 和 $n^{-1 / 2} \sum_{i=1}^{n} B\left\{\boldsymbol{\beta}_{0}(\tau)\right\}^{-1} \phi\left\{\iota_{i}(\tau)\right\}$ 是渐近等价的. 因而,

$$
K(\tau)+o_{\left(\nu, \tau_{U}\right]}(1)=n^{-1 / 2} \sum_{i=1}^{n}\left[q\left(\boldsymbol{Z}_{i}\right) M_{i}\left(\tau ; \boldsymbol{\beta}_{0}\right)-\psi\left(B\left\{\boldsymbol{\beta}_{0}(\tau)\right\}^{-1} \phi\left\{\iota_{i}(\tau)\right\}\right)\right]=n^{-1 / 2} \sum_{i=1}^{n} \xi_{i}(\tau) .
$$

根据 $M_{i}\left(\tau, \boldsymbol{\beta}_{0}\right)$ 的零均值特性, 且 $\mathrm{E}\left\{\iota_{i}(\tau)\right\}=0$, 对于 $\tau \in\left(0, \tau_{U}\right]$, 我们有 $\mathrm{E}\left\{\xi_{i}(\tau)\right\}=0$. 根据 Donsker 定理, $K(\tau)$ 弱收玫到一个零均值, 协方差函数为 $\Omega(s, t)=\mathrm{E}\left\{\xi_{i}(s) \xi_{i}(t)^{\mathrm{T}}\right\}$ 的 Gauss 过程.

接下来, 我们考虑 $K^{*}(\cdot)$ 的渐近性质. 从 $K^{*}(\tau)$ 的定义、方程 (C.1) 和 (C.2), 我们得到

$$
K^{*}(\tau)=n^{-1 / 2} \sum_{i=1}^{n}\left(1-\zeta_{i}\right)\left[q\left(\boldsymbol{Z}_{i}\right) M_{i}(\tau ; \hat{\boldsymbol{\beta}})-\psi\left(B\{\hat{\boldsymbol{\beta}}(\tau)\}^{-1} \phi\left\{\hat{\iota}_{i}(\tau)\right\}\right)\right]+o_{\left(\nu, \tau_{U}\right]}(1)
$$




$$
=n^{-1 / 2} \sum_{i=1}^{n}\left(1-\zeta_{i}\right) \hat{\xi}_{i}(\tau)+o_{\left(\nu, \tau_{U}\right]}(1)
$$

在给定 $\left\{A_{i}, Y_{i}, \delta_{i}, \boldsymbol{Z}_{i}\right\}_{i=1}^{n}$ 的条件下, $K^{*}(\tau)$ 弱收敛到一个均值为 0 、协方差矩阵 $\hat{\Omega}(s, t)=\mathrm{E}\left\{\hat{\xi}_{i}(s) \hat{\xi}_{i}(t)^{\mathrm{T}}\right\}$ 的 Gauss 过程. 当给定的模型成立时, 对于 $s, t \in\left[\nu, \tau_{U}\right], \hat{\Omega}(s, t)$ 几乎处处收敛到 $\Omega(s, t)$.

\title{
Estimating equation methods for quantile regression with length-biased and right-censored data
}

\author{
MA HuiJuan, FAN CaiYun \& ZHOU Yong
}

Abstract In this paper, we first develop the quantile regression approach for survival data arising from lengthbiased sampling, where the survival times are left-truncated by uniformly distributed random truncated times. As a special case of the left-truncated and right-censored (LTRC) data, length-biased right-censored (LBRC) data supports more information than LTRC data. When we apply the general methods to LBRC data, the resulting estimators may be inefficient because they do not exploit the special structure in LBRC data. In order to improve efficiency, we then develop a composite quantile regression approach for LBRC data, which do not need to estimate the distribution of censoring time. The proposed estimating equations lead to a simple algorithm that involves minimizations of $L_{1}$ type convex functions. We establish uniform consistency and weak convergence of the resultant estimators using empirical process and stochastic integral techniques. Simulation studies are conducted to evaluate the finite sample performance of the proposed methods. A real data example is also given.

Keywords left-truncated, right-censored, length-biased sampling, quantile regression, empirical process MSC(2010) 62N02

doi: $10.1360 / 012015-36$ 